\title{
CONVERGENCE OF CELL BASED FINITE VOLUME DISCRETIZATIONS FOR PROBLEMS OF CONTROL IN THE CONDUCTION COEFFICIENTS*
}

\author{
Anton Evgrafov $^{1}$, Misha Marie Gregersen ${ }^{1}$ and Mads Peter Sørensen ${ }^{1}$
}

\begin{abstract}
We present a convergence analysis of a cell-based finite volume (FV) discretization scheme applied to a problem of control in the coefficients of a generalized Laplace equation modelling, for example, a steady state heat conduction. Such problems arise in applications dealing with geometric optimal design, in particular shape and topology optimization, and are most often solved numerically utilizing a finite element approach. Within the FV framework for control in the coefficients problems the main difficulty we face is the need to analyze the convergence of fluxes defined on the faces of cells, whereas the convergence of the coefficients happens only with respect to the "volumetric" Lebesgue measure. Additionally, depending on whether the stationarity conditions are stated for the discretized or the original continuous problem, two distinct concepts of stationarity at a discrete level arise. We provide characterizations of limit points, with respect to FV mesh size, of globally optimal solutions and two types of stationary points to the discretized problems. We illustrate the practical behaviour of our cell-based FV discretization algorithm on a numerical example.
\end{abstract}

Mathematics Subject Classification. 65N08, 65N12, 49M05, 49M25.

Received November 22, 2010. Revised March 4, 2011.

Published online June 28, 2011.

\section{INTRODUCTION AND MOTIVATION}

Utilizing control in the coefficients of partial differential equations (PDEs) for the purpose of optimal design, or topology optimization, is a well established technique in both academia and industry; see for example an early paper by Bendsøe and Kikuchi [9] or more recent monographs [4,10] and the references therein. Advantages of using control in the coefficients for optimal design purposes include the flexibility of the induced parametrization of the design space that allows optimization algorithms to efficiently explore it, the ease of integration with existing computational modelling codes in a variety of application areas, and the simplicity and efficiency of sensitivity analyses. Control in the coefficients has already been successfully used in a variety of application domains including structural, solid, and fluid mechanics, as well as transport and multi-physics applications; and at a variety of length-scales, including macro-, micro-, and nano-scales [10]. As the complexity of the PDEs governing the underlying physical phenomena increases, it becomes important to utilize robust PDE solvers within control in the coefficients framework.

\footnotetext{
Keywords and phrases. Topology optimization, finite volume methods.

* The authors acknowledge the support of the European Commission in the project "Fluid Optimisation Workflows for Highly Effective Automotive Development Processes" under theme SST.2007-RTD-1: Competitive product development.

${ }^{1}$ Department of Mathematics, Technical University of Denmark, 2800 Kgs. Lyngby, Denmark. a.evgrafov@mat.dtu.dk
} 
Finite volume methods (FVMs) constitute a very mature and versatile technique for discretizing partial differential equations in the form of conservation laws of varying types: elliptic, parabolic, hyperbolic, and mixed [21]. FVMs are known for the simplicity of implementation, their local conservation properties, and the ease of coupling various PDEs in a multi-physics setting. Presently, FVMs represent a standard choice of discretization within engineering communities dealing with computational fluid dynamics, transport, and convection-reaction problems. This is particularly important as control in the coefficients is being applied in these application domains, see for example $[2,6,12,16-20,27,28,30,31,33-37,40-42,46]$. Among various flavours of FVMs, cell based approaches, where all variables are associated only with cell centers, are particularly attractive. They bring further simplifications to the implementation of such a scheme, as all involved PDEs on a given domain are discretized using the same and the lowest possible number of degrees of freedom associated with a given mesh.

In spite of their attractiveness FVMs have seen very little adoption within the topology optimization community, where the absolute majority of numerical computations is done using finite element methods (FEMs). The few exceptions include $[30,33,36,37]$ in the context of topology optimization within computational fluid dynamics, and [26] where a control of a steady state heat conduction boundary value problem (BVP) is treated numerically. Despite these recent efforts, we have not even scratched the surface as far as our understanding of the interplay between the control in the coefficients and FVMs is concerned.

To support our previous statement and illustrate the differences between FVM and FEM discretizations of control in the coefficients problems, let us informally consider the following steady-state conservation law:

$$
\operatorname{div}\left(F\left(u, \nabla u ; \alpha_{F}\right)\right)+s\left(u ; \alpha_{s}\right)=0, \quad \text { in } \Omega .
$$

In (1.1), $\Omega$ is a polygonal domain in $\mathbb{R}^{d}, d \in \mathbb{N}, u: \Omega \rightarrow \mathbb{R}$ is a sought solution to the conservation law, $F: \mathbb{R} \times \mathbb{R}^{d} \times \mathbb{R} \rightarrow \mathbb{R}^{d}$ and $s: \mathbb{R} \times \mathbb{R} \rightarrow \mathbb{R}$ define the flux and the source terms of the law, and finally $\alpha_{F}: \Omega \rightarrow \mathbb{R}, \alpha_{s}: \Omega \rightarrow \mathbb{R}$ are controllable coefficients entering the flux and the source terms. Within the framework of conforming FEM discretizations, we multiply (1.1) with a suitable test function $v$ coming from a finite-dimensional subspace of the solution space for $u$, and integrate the flux term by parts (in this informal discussion we assume that such an integration is warranted) thus obtaining the variational formulation

$$
\int_{\Omega} F\left(u, \nabla u ; \alpha_{F}\right) \cdot \nabla v \mathrm{~d} x=\int_{\Omega} s\left(u ; \alpha_{s}\right) v \mathrm{~d} x+\int_{\partial \Omega} F\left(u, \nabla u ; \alpha_{F}\right) \cdot \mathbf{n} v \mathrm{~d} x, \quad \forall v,
$$

where $\mathbf{n}: \partial \Omega \rightarrow \mathbb{R}^{d}$ is the outwards facing normal for $\Omega$. The most common situation is that owing to the boundary conditions on $\partial \Omega$ either the normal flux $\mathbf{n} \cdot F\left(u, \nabla u ; \alpha_{F}\right)$ is known as an expression independent from $\alpha_{F}$ (typical Neumann, Robin boundary conditions) or $v=0$ (Dirichlet boundary conditions). Therefore, within the conforming FEM discretization approach normally only volume integrals depend on the control coefficients. The behaviour of the solution $u$ as a function of $\left(\alpha_{F}, \alpha_{s}\right)$ is well understood in this case: usually we only need to demand pointwise convergence of $\alpha_{F}$ and even weaker convergence of $\alpha_{s}$ to achieve some form of continuous change in $u_{\alpha_{F}, \alpha_{s}}$. Intuitively, one can interpret this behaviour as follows: small changes in $\left(\alpha_{F}, \alpha_{s}\right)$ generate small changes in the volume integrals involved in (1.1) and subsequently (as a consequence of continuity and stability properties of the associated bilinear forms) small changes in $u_{\alpha_{F}, \alpha_{s}}$, see Figure 1.

Let us now apply an FVM discretization to (1.1). We decompose $\Omega$ into disjoint convex polygonal control volumes $K \in \mathcal{T}$, integrate over each control volume while applying Gauss-Ostrogradsky theorem to the flux term; we finally arrive at the following formulation:

$$
\int_{\partial K} F\left(u, \nabla u ; \alpha_{F}\right) \cdot \mathbf{n} \mathrm{d} x+\int_{K} s\left(u ; \alpha_{s}\right) \mathrm{d} x=0, \quad \forall K \in \mathcal{T},
$$

where $\mathbf{n}$ is an outwards directed normal for the control volume $K$. Suddenly we see the distinct rôles which $\alpha_{F}$ and $\alpha_{s}$ play in the FVM discretization: $\alpha_{F}$ is involved in the boundary integrals with respect to $d$-1-dimensional Lebesgue measure, whereas $\alpha_{s}$ as in (1.2) only enters volume integrals w.r.t. $d$-dimensional Lebesgue measure. 

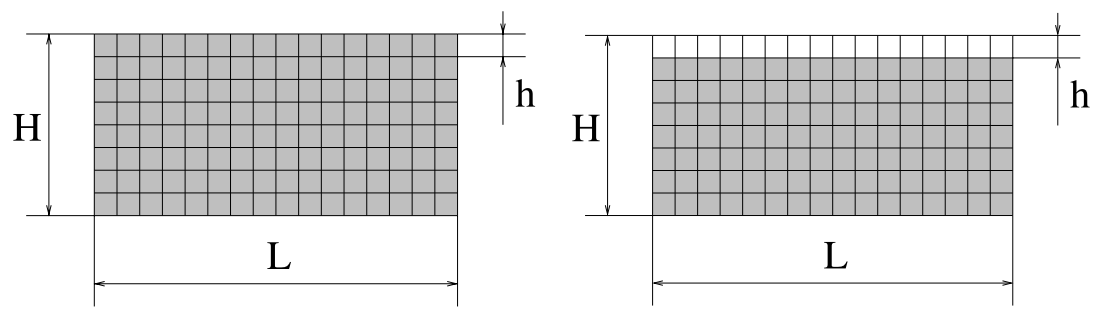

Figure 1. Modifying a control coefficient $\alpha_{F}$ or $\alpha_{s}$ on a small set of $d$-dimensional Lebesgue measure $h L(h \rightarrow 0)$ introduces a small perturbation of the order $O(h L \Delta \alpha)$ to the volume integrals involved in the conforming FEM formulation (1.2). However, the same change to $\alpha_{F}$ introduces a finite perturbation of the order $O(L \Delta \alpha)$ to the boundary integrals involved in the FVM discretization (1.3).

Of course, small w.r.t. $d$-dimensional Lebesgue measure perturbations to $\alpha_{F}$ may introduce very large changes to the flux integrals involved in (1.3), see Figure 1. Therefore, a study of the interplay between the FVM discretization and the control in the coefficients approach, where the control coefficient enters the flux integral, is warranted. In the present paper we conduct such a study for a simple model problem formally described in Section 2 .

We now put the results obtained in this study into the proper perspective.

(i) To the best of our knowledge, this is the first rigorous study of convergence of FVM discretizations of control in the coefficients problems with respect to the mesh refinement. We provide characterizations of the limit points of both globally optimal solutions and two kinds of stationary points to the discretized problems, see Theorems 4.7, 5.3, and 5.5. Such an analysis is lacking in the works utilizing FVM discretizations of control in the coefficients problems [26,30,33,36,37]. For conforming FEM approximations a related study, which however deals only with globally optimal solutions, is [39].

(ii) We provide an alternative proof of convergence of FVM discretization of the diffusion term with a discontinuous coefficient without approximating it with a sequence of $C^{1}$ coefficients first, $c f$. Lemmas 4.3 and [22], Lemma 2.1 .

(iii) As far as numerical methods are concerned, this work also differs from the few numerical studies of FVM discretizations of control in the coefficients problems. Compared to [30,33,36,37], which involve only control coefficients $\alpha_{s}$ in the notation we used, our study concentrates on the flux control coefficient $\alpha_{F}$. Also, in addition to the continuous adjoint approach employed in the cited works we treat the problem with both continuous and discrete adjoint approaches, see Section 5. Compared to [26], which treats the same physical problem as the present paper using a vertex-based FVM scheme on a structured grid and a discrete adjoint approach, we employ cell-based FVM discretizations; we do not assume that the underlying meshes are structured (see Sect. 3); and finally, we employ both continuous and discrete adjoint approaches.

The remainder of this paper is organized as follows. In Section 2 we describe the model control in the coefficients problem, and more precisely the properties we expect numerical approximations to this problem should possess. Section 3 describes in great detail the FVM discretization applied to the model problem; most of the information provided in this section can be found in $[21,23]$ and is present here to keep the paper self-contained. Section 4 is dedicated to studying the continuity properties of the implicit coefficient-to-solution (design-to-state) mapping defined by the FVM discretization with respect to the mesh refinement. We utilize this continuity for characterizing the limit points of globally optimal solutions (in Sect. 4) and two kinds of stationary points (in Sect. 5) of the FVM-discretized model control in the coefficients problem. In Section 6 we illustrate the practical behavior of our cell-based FVM discretization algorithm. We finish the paper with a brief discussion and possible further research directions. 
To conclude this introductory section we mention that the computational approach, which we follow in this paper, is based on discretizing control in the coefficients problems first and then computing optimal solutions/stationary points at a discrete level. An alternative approach, considered for example in [45], is to formulate and analyze an optimization algorithm at a continuous level. However, since in practice one would still have to discretize the algorithm at some point, the analysis presented in this manuscript remains relevant. In fact, the results presented in Section 5.1 may well be interpreted as an application of such "optimize-thendiscretize" ideology.

\section{Model Continuous PRoblem}

\subsection{Problem formulation}

For simplicity, we limit our analysis to a mathematical program involving an implicit mapping given by a homogeneous Dirichlet boundary value problem (BVP) for an isotropic but heterogeneous steady state diffusion operator; we note that this can be extended to more general boundary conditions and elliptic operators [22,25].

Namely, a typical setup found in the engineering optimization problems is as follows. Let $\Omega$ be polygonal domain in $\mathbb{R}^{d}, d \in \mathbb{N}$ with a boundary $\partial \Omega=\Gamma$. Further, let $G \subset \mathcal{M}(\Omega)$ be a given subset of measurable functions $\gamma: \Omega \rightarrow[0,1]$ closed with respect to a.e. convergence on $\Omega$. We will be interested in numerically approximating locally and/or globally optimal solutions to the following minimization problem:

$$
\begin{array}{ll}
\text { minimize } & c(\gamma), \\
\text { subject to } & \gamma \in G .
\end{array}
$$

We will mostly be interested in approximating locally optimal solutions to (2.1); however, we also provide the analysis of convergence of approximations to globally optimal solutions to (2.1) in Theorem 4.7. To ensure the existence of globally optimal solutions to (2.1), in Theorem 4.7 we will make an additional assumption about sequential compactness of $G$ with respect to a.e. convergence in $\Omega$. The latter property is for example implied by the compactness of $G$ in $L^{1}(\Omega)$, a situation found in practical applications of control in the coefficients [39].

In (2.1), the (reduced) cost functional $c: G \rightarrow \mathbb{R}$ is defined implicitly as follows:

$$
c(\gamma)=\int_{\Omega} \mathcal{C}\left(x, \gamma(x), u_{\gamma}(x), \nabla u_{\gamma}(x)\right) \mathrm{d} x,
$$

where $u_{\gamma} \in H_{0}^{1}(\Omega)$ is the unique solution of the BVP

$$
-\operatorname{div}\left[\alpha(\gamma) \nabla u_{\gamma}\right]=f,
$$

$f \in L^{2}(\Omega), \alpha:[0,1] \rightarrow[\underline{\alpha}, \bar{\alpha}]$ is a continuous mapping of the "design space" $G$ to the space of diffusion coefficients, $0<\underline{\alpha} \leq \bar{\alpha}<+\infty$. The cost function $\mathcal{C}: \Omega \times[0,1] \times \mathbb{R} \times \mathbb{R}^{d} \rightarrow \mathbb{R}$ is assumed to be measurable with respect to its first argument, and continuous with respect to its last three arguments. In order to warrant the evaluation of the integral in $(2.2)$ we assume that $\mathcal{C}$ satisfies the quadratic growth condition

$$
|\mathcal{C}(x, \gamma, u, z)| \leq C\left(1+|u|^{2}+|z|^{2}\right), \quad \forall(x, \gamma, u, z) \in \Omega \times[0,1] \times \mathbb{R}^{1+d},
$$

for some constant $C>0$. By a variant of the dominated convergence theorem (see, e.g., [15], Thm. 4, p. 21) the integral of $\mathcal{C}$ is continuous with respect to poinwise a.e. convergence of $\gamma$ and strong $L^{2}(\Omega)$-convergence of $u$ and $z$ :

$$
\begin{aligned}
\lim _{n \rightarrow \infty} \int_{\Omega} \mathcal{C}\left(x, \gamma_{n}(x), u_{n}(x), z_{n}(x)\right) \mathrm{d} x=\int_{\Omega} \mathcal{C}(x, \gamma(x), u(x), z(x)) \mathrm{d} x \\
\quad \forall\left(\gamma_{n}, \gamma, u_{n}, u, z_{n}, z\right) \in G^{2} \times\left[L^{2}(\Omega)\right]^{2+2 d}, n=1,2, \ldots: \\
\quad \lim _{n \rightarrow \infty} \gamma_{n}(x)=\gamma(x), \text { a.e. in } \Omega, \lim _{n \rightarrow \infty}\left\|\left(u_{n}, z_{n}\right)-(u, z)\right\|_{\left[L^{2}(\Omega)\right]^{1+d}}=0 .
\end{aligned}
$$


In what follows, it will be more convenient to characterize $u_{\gamma}$ as the unique function in $H_{0}^{1}(\Omega)$ satisfying the following variational problem:

$$
a_{\gamma}\left(u_{\gamma}, v\right)=\ell(v), \quad \forall v \in H_{0}^{1}(\Omega)
$$

where $a_{\gamma}:\left[H_{0}^{1}(\Omega)\right]^{2} \rightarrow \mathbb{R}$ is a continuous and coercive symmetric $\gamma$-parametric bilinear form and $\ell \in\left[H_{0}^{1}(\Omega)\right]^{*}$ is a linear continuous functional defined via

$$
a_{\gamma}(u, v)=\int_{\Omega} \alpha(\gamma(x)) \nabla u(x) \cdot \nabla v(x) \mathrm{d} x, \quad \ell(v)=\int_{\Omega} f(x) v(x) \mathrm{d} x .
$$

\subsection{Continuity of the coefficient-to-solution mapping}

One characteristic property of the minimization problem (2.1) is that it involves an implicit mapping (coefficient-to-solution mapping) $G \ni \gamma \mapsto u_{\gamma} \in H_{0}^{1}(\Omega)$. A successful approximation scheme for (2.1) should therefore mimic certain properties of this mapping. For example, in the case of our model BVP given by (2.6) the coefficient-to-solution mapping is known to enjoy the following property (see [7], Thm. 16.4.2):

Proposition 2.1. Let $\gamma^{(n)} \in G, n=1,2, \ldots$ be a sequence of measurable functions, and further let $u^{(n)} \in H_{0}^{1}(\Omega)$ be the sequence of solutions to (2.6) with $\gamma=\gamma^{(n)}$. Assume that $\gamma^{(n)} \rightarrow \widehat{\gamma}$, a.e. in $\Omega$, and let $\widehat{u} \in H_{0}^{1}(\Omega)$ be the solution to (2.6) with $\gamma=\widehat{\gamma}$. Then $u^{(n)} \rightarrow \widehat{u}$ strongly in $H_{0}^{1}(\Omega)$.

To stress the importance of Proposition 2.1, we note that it is a key argument within the framework of the direct method of calculus of variations [13] applied to (2.1). Namely, combining this result with continuity (2.5) for $\mathcal{C}$ and sequential compactness assumptions for $G$ with respect to a.e.-convergence in $\Omega$, one easily obtains existence of optimal solutions to (2.1). Whereas such a compactness of $G$ is a rather stringent assumption, it is nevertheless verified when the variation of functions in $G$ is uniformly bounded (see, e.g., [15], Thm. 4, p. 176), a condition which is known as the "perimeter constraint" in the optimal design literature $[5,24,29,39]$.

Similarly, in order to establish convergence of numerical approximations to (2.1) it is imperative that an appropriate discrete version of Proposition 2.1 holds. For example, for conforming finite element approximations to (2.1) the following result is known (see [39], Lem. 2.2):

Proposition 2.2. Let $\gamma^{(n)}, n=1,2, \ldots$ and $\widehat{\gamma}, \widehat{u}$ be as in Proposition 2.1. Consider a sequence of closed subspaces $V^{(n)}$ of $H_{0}^{1}(\Omega)$ (for example, standard piece-wise polynomial subspaces used in $H^{1}$-conforming finite element approximations) such that for every $\phi \in H_{0}^{1}(\Omega)$ it holds that

$$
\lim _{n \rightarrow \infty} \inf _{v^{(n)} \in V^{(n)}}\left\|\phi-v^{(n)}\right\|_{H^{1}(\Omega)}=0
$$

Let $u^{(n)} \in V^{(n)}$ be a solution to the approximate variational problem, that is $a_{\gamma^{(n)}}\left(u^{(n)}, v\right)=\ell(v)$ for every $v \in V^{(n)}$. Then $u^{(n)} \rightarrow \widehat{u}$ strongly in $H_{0}^{1}(\Omega)$.

We note that the discrete version of the result is in fact stronger than its continuous counterpart: indeed, Proposition 2.1 is just a special case of Proposition 2.2 recovered by setting $V^{(n)}=H_{0}^{1}(\Omega)$.

The significance of results such as Proposition 2.2 stems from the fact that they serve as basic building blocks for establishing convergence of numerical approximations to problems of control in the coefficients. In the cited work [39] as well as in the overwhelming majority of works on topology optimization [10], a conforming finite element method was used to discretize the underlying governing partial differential equations. One of the goals of the present paper is to establish a result analogous to Proposition 2.2, in which the approximation to (2.3) is constructed using a finite volume method. In particular, the spaces $V^{(n)}$ in this case are spaces of piecewise constant functions. Such approximation spaces are non-conforming, that is, they are not subspaces of $H_{0}^{1}(\Omega)$ any longer. In addition, defining the gradient pointwise on these spaces one can never satisfy the approximation condition (2.8) of Proposition 2.2. Nevertheless, recent advances in discrete functional analysis, such as the discrete Rellich-Kondrachov theorem, construction and convergence of discrete gradients (see for example $[14,23]$ ) allow us to carry out such a task. 


\subsection{Optimality conditions}

Globally optimal solutions to (2.1), even when they exist, are of little practical interest outside of an academic environment, because within most numerical approaches we can only generate solutions verifying certain optimality conditions. To fix the ideas, we assume the following setup:

(i) The function $\alpha \in C^{1}([0,1])$;

(ii) The cost function $\mathcal{C}$ is independent from $\nabla u$ (that is, $\mathcal{C}=\mathcal{C}(x, \gamma, u)$ ) and is continuously differentiable with respect to $\gamma$ and $u$. Partial derivatives $\partial \mathcal{C} / \partial \gamma$ and $\partial \mathcal{C} / \partial u$ verify the growth conditions:

$$
\begin{array}{ll}
|\partial \mathcal{C} / \partial \gamma(x, \gamma, u)| \leq C\left(1+|u|^{2}\right), \quad \forall(x, \gamma, u) \in \Omega \times[0,1] \times \mathbb{R}, \\
|\partial \mathcal{C} / \partial u(x, \gamma, u)| \leq C(1+|u|), \quad \forall(x, \gamma, u) \in \Omega \times[0,1] \times \mathbb{R} .
\end{array}
$$

Similarly to (2.5), we get the following forms of continuity:

$$
\begin{gathered}
\lim _{n \rightarrow \infty} \int_{\Omega} \frac{\partial \mathcal{C}}{\partial \gamma}\left(x, \gamma_{n}(x), u_{n}(x)\right) \delta_{n}(x) \mathrm{d} x=\int_{\Omega} \frac{\partial \mathcal{C}}{\partial \gamma}(x, \gamma(x), u(x)) \delta(x) \mathrm{d} x \\
\lim _{n \rightarrow \infty} \int_{\Omega} \frac{\partial \mathcal{C}}{\partial u}\left(x, \gamma_{n}(x), u_{n}(x)\right) \lambda(x) \mathrm{d} x=\int_{\Omega} \frac{\partial \mathcal{C}}{\partial u}(x, \gamma(x), u(x)) \lambda(x) \mathrm{d} x \\
\forall\left(\gamma_{n}, \gamma, \delta_{n}, \delta, u_{n}, u, \lambda\right) \in G^{2} \times\left[L^{\infty}(\Omega)\right]^{2} \times\left[L^{2}(\Omega)\right]^{3}, n=1,2, \ldots: \\
\quad \lim _{n \rightarrow \infty}\left[\gamma_{n}(x), \delta_{n}(x)\right]=[\gamma(x), \delta(x)], \text { a.e. in } \Omega, \\
\quad \sup _{n}\left\|\delta_{n}\right\|_{L^{\infty}(\Omega)}<+\infty, \lim _{n \rightarrow \infty}\left\|u_{n}-u\right\|_{L^{2}(\Omega)}=0 .
\end{gathered}
$$

(iii) Finally, for simplicity we assume that the design space $G$ is convex (although most developments should extend easily to sets $G$ given by smooth constraints under appropriate constraint qualifications).

Under condition (i) we can utilize the implicit function theorem (see e.g., [47], Thm. 4.E) to infer that the coefficient-to-solution mapping is Fréchet differentiable, when $G$ is equipped with $L^{\infty}(\Omega)$-norm. Owing to the condition (ii) and the chain rule (see e.g., [47], Thm. 4.D), the reduced cost function $c$ is Fréchet differentiable as well. Its derivative in the direction $\delta \in L^{\infty}(\Omega)$, as can be easily verified, is given by the expression:

$$
D c(\gamma ; \delta)=\int_{\Omega} \frac{\partial \mathcal{C}}{\partial \gamma}\left(x, \gamma, u_{\gamma}\right) \delta \mathrm{d} x+\int_{\Omega} \alpha^{\prime}(\gamma) \delta \nabla u_{\gamma} \cdot \nabla \lambda_{\gamma} \mathrm{d} x
$$

where $\lambda_{\gamma} \in H_{0}^{1}(\Omega)$ is the unique solution to the adjoint problem

$$
a_{\gamma}\left(v, \lambda_{\gamma}\right)=\ell_{\mathrm{adj}, \gamma, u_{\gamma}}(v), \quad \forall v \in H_{0}^{1}(\Omega)
$$

with $\ell_{\mathrm{adj}, \gamma, u_{\gamma}} \in\left[H_{0}^{1}(\Omega)\right]^{*}$ given by

$$
\ell_{\operatorname{adj}, \gamma, u_{\gamma}}(v)=-\int_{\Omega} \frac{\partial \mathcal{C}}{\partial u}\left(x, \gamma, u_{\gamma}\right) v \mathrm{~d} x .
$$

Finally, owing to the assumption (iii), every locally (with respect to $L^{\infty}(\Omega)$-norm) optimal solution $\gamma^{*} \in G$ to (2.1) must satisfy the following variational inequality (see, e.g., [11], Lem. 3.7):

$$
D c\left(\gamma^{*} ; \gamma-\gamma^{*}\right) \geq 0, \quad \forall \gamma \in G
$$

In this paper we will study whether limit points of sequences of solutions verifying certain discrete versions of stationarity can also be expected to satisfy (2.14). 


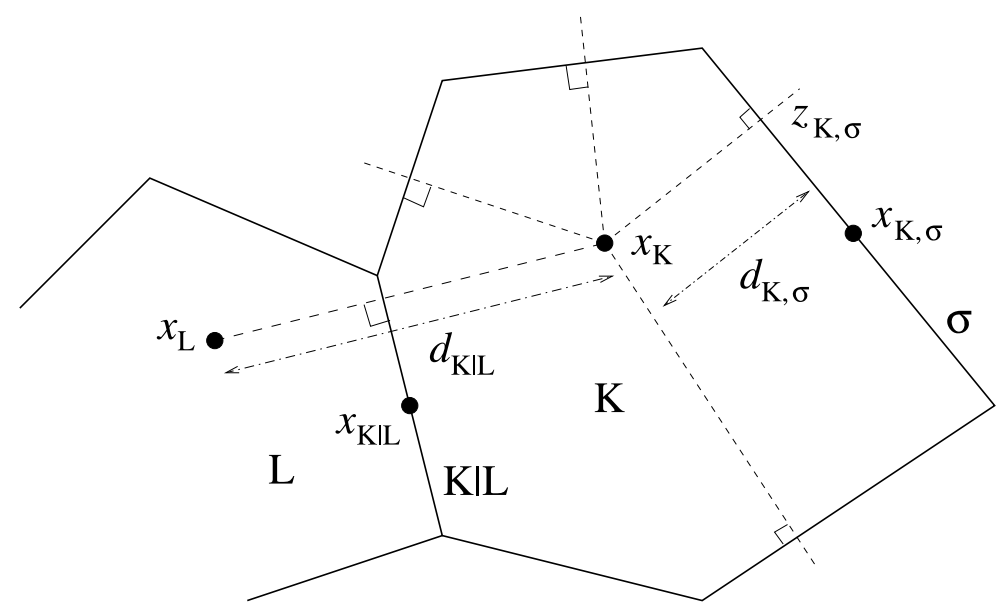

Figure 2. Admissible finite volume discretization with associated notation for a control volume in two dimensions.

\section{Cell-Centered finite volume approximations}

\subsection{Admissible finite volume discretizations}

We utilize finite volume method for discretizing the direct problem (2.3), the adjoint problem (2.12), and finally the control in the coefficients problem (2.1). We employ the notation and the approach of [21,23], which we introduce in this section to keep the paper self-contained.

An admissible finite volume discretization $\mathcal{D}$ of $\Omega$, in the sense of [21], also see Figure 2 , is a triple $(\mathcal{T}, \mathcal{E}, \mathcal{P})$, where

(i) $\mathcal{T}$ is a finite collection of non-empty open disjoint convex polygonal subsets of $\Omega$ (also known as the "control volumes") such that $\bar{\Omega}=\cup_{K \in \mathcal{T}} \bar{K}$. For each $K \in \mathcal{T}, \partial K$ denotes the boundary of $K$ and $m(K)$ is the $d$-dimensional Lebesgue measure of $K$.

(ii) $\mathcal{E}$ is a finite family of disjoint subsets of $\bar{\Omega}$ (the edges in $2 \mathrm{D}$, or (hyper-)faces of the mesh in $d \geq 3$ ). For every $\sigma \in \mathcal{E}$, there is a $d$-1-dimensional hyperplane $E \subset \mathbb{R}^{d}$ and $K \in \mathcal{T}$ such that $\bar{\sigma}=\partial K \cap E$ and $\sigma$ is a non-empty open subset of $E . m(\sigma)$ will denote the $d$-1-dimensional Lebesgue measure of $\sigma$. For every $K \in \mathcal{T}$, there is a subset $\mathcal{E}_{K} \subseteq \mathcal{E}$ such that $\partial K=\cup_{\sigma \in \mathcal{E}_{K}} \bar{\sigma}$. As a result of these assumptions, every $\sigma \in \mathcal{E}$ is either a subset of $\partial \Omega$, or a face common to two control volumes $K, L \in \mathcal{T}: \bar{\sigma}=\bar{K} \cap \bar{L}$. In the latter case we will denote the edge with $K \mid L$. The set of boundary edges will be denoted with $\mathcal{E}_{\text {ext }}$ and the set of interior edges is $\mathcal{E}_{\text {int }}$. For every $\sigma \in \mathcal{E}$ we define its center:

$$
x_{\sigma}=\frac{1}{m(\sigma)} \int_{\sigma} x \mathrm{~d} x
$$

(iii) $\mathcal{P}=\left\{x_{K} \mid K \in \mathcal{T}\right\}$ is a finite collection of points in $\Omega$, such that for every $K \in \mathcal{T}$ it holds that $x_{K} \in K$. For every $K \mid L \in \mathcal{E}_{\text {int }}$ we assume that $x_{K}-x_{L}$ is orthogonal to $K \mid L$. Finally, let $z_{K, \sigma}$ be an orthogonal projection of $x_{K}$ onto $\sigma \in \mathcal{E}_{K}$, then $z_{K, \sigma} \in \sigma$ for every $\sigma \subseteq \partial \Omega$. We denote the distance between $x_{K}$ and $z_{K, \sigma}$ with $d_{K, \sigma}$, and the distance between $x_{K}$ and $x_{L}$ with $d_{K \mid L}$.

For every discretization $\mathcal{D}$ we measure its size with

$$
h_{\mathcal{D}}=\sup _{K \in \mathcal{T}} \operatorname{diam}(K),
$$


and its regularity with

$$
\theta_{\mathcal{D}}=\inf _{K \in \mathcal{T}, \sigma \in \mathcal{E}_{K}} \frac{d_{K, \sigma}}{\operatorname{diam}(K)}
$$

For every $K \in \mathcal{T}$ we denote by $\mathcal{N}(K) \subset \mathcal{T}$ the control volumes having a common edge with $K$ (not including $K$ itself). For all $K \mid L \in \mathcal{E}_{\text {int }}$ we denote $\mathbf{n}_{K \mid L}=d_{K \mid L}^{-1}\left(x_{K}-x_{L}\right)$ the unit normal to $K \mid L$ directed from $L$ to $K$. For every $K \in \mathcal{T}, \sigma \in \mathcal{E}_{K}$, we write $\mathbf{n}_{K, \sigma}=d_{K, \sigma}^{-1}\left(z_{K, \sigma}-x_{K}\right)$ for a unit outward normal for $K$ on $\sigma$. We also set

$$
\tau_{K \mid L}=\frac{m(K \mid L)}{d_{K \mid L}}
$$

Similarly, for every $K \in \mathcal{T}$ and $\sigma \in \mathcal{E}_{K}$, we set

$$
\tau_{K, \sigma}=\frac{m(\sigma)}{d_{K, \sigma}}
$$

For $\sigma \in \mathcal{E}_{\text {ext }}$, let $K \in \mathcal{T}$ be such that $\sigma \in \mathcal{E}_{K}$; then we set $\tau_{\sigma}=\tau_{K, \sigma}$.

\subsection{Cell centered approximation of (2.3)}

Given an admissible discretization $\mathcal{D}=(\mathcal{T}, \mathcal{E}, \mathcal{P})$ we are ready to construct approximations of the direct problem (2.3). Let $H_{\mathcal{D}}(\Omega)$ be a set of functions from $\Omega$ to $\mathbb{R}$ constant on every control volume $K \in \mathcal{T}$; this is going to be an approximation space for solutions to the boundary value problem (2.3). Similarly, let $G_{\mathcal{D}}$ be a non-empty closed set approximating $G$ with functions which are constant on every control volume and also have values in $[0,1]$. We will often write $\alpha_{K}$ in place of $\alpha\left(\gamma_{K}\right), \gamma \in G_{\mathcal{D}}$. We will also need the interpolation operator $P_{\mathcal{D}}:\left[C^{0}(\Omega)\right]^{n} \rightarrow\left[H_{\mathcal{D}}(\Omega)\right]^{n}$ given by $\left[P_{\mathcal{D}} \phi\right](x)=\phi\left(x_{K}\right)$, for $x \in K$.

After integrating the equation (2.3) on every control volume $K \in \mathcal{T}$ and utilizing Gauss-Ostrogradsky theorem, we end up with

$$
\sum_{\sigma \in \mathcal{E}_{K}} F_{K, \sigma}(\alpha, u)=m(K) f_{K}, \quad \forall K \in \mathcal{T},
$$

where $f_{K}=m(K)^{-1} \int_{K} f(x) \mathrm{d} x$, and $F_{K, \sigma}: H_{\mathcal{D}}(\Omega) \times H_{\mathcal{D}}(\Omega) \rightarrow \mathbb{R}$ is an approximation of the normal flux $\alpha \nabla u \cdot \mathbf{n}$ through the edge $\sigma$. It is common to use the following central difference approximation of the diffusive fluxes:

$$
F_{K, \sigma}(\alpha, u)= \begin{cases}-\alpha_{K \mid L} \tau_{K \mid L}\left(u_{L}-u_{K}\right), & \text { if } \sigma=K \mid L \in \mathcal{E}_{\text {int }}, \\ -\alpha_{K} \tau_{\sigma}\left(-u_{K}\right), & \text { if } \sigma \in \mathcal{E}_{\text {ext }},\end{cases}
$$

where

$$
\alpha_{K \mid L}=\left(d_{K, K \mid L} \alpha_{K}+d_{L, K \mid L} \alpha_{L}\right) / d_{K \mid L},
$$

is an approximation of the diffusion coefficient across the edge $K \mid L$. Note that in the engineering practice of finite volume methods for diffusion problems with discontinuous coefficients, particularly on coarse meshes, weighted harmonic averaging of $\alpha_{K}, \alpha_{L}$ is often successfully employed in place of arithmetic averaging (3.3). However, a convergence proof for such a scheme remains an open problem [22]; therefore, throughout the remainder of the paper we assume that weighted arithmetic averaging (3.3) is used in the definition of fluxes.

While equations (3.1)-(3.3) provide a complete description of the finite-volume method for (2.3), for the purpose of the convergence analysis it is more convenient to rewrite (3.1) in the variational form, see [21,23]. For $\gamma \in G_{\mathcal{D}}$ we introduce an $\gamma$-parametric bilinear form $a_{\gamma, \mathcal{D}}:\left[H_{\mathcal{D}}(\Omega)\right]^{2} \rightarrow \mathbb{R}$ and a linear functional $\ell_{\mathcal{D}} \in\left[H_{\mathcal{D}}(\Omega)\right]^{*}:$

$$
\begin{aligned}
a_{\gamma, \mathcal{D}}(u, v) & =\sum_{K \mid L \in \mathcal{E}_{\mathrm{int}}} \alpha_{K \mid L} \tau_{K \mid L}\left(u_{L}-u_{K}\right)\left(v_{L}-v_{K}\right)+\sum_{\sigma \in \mathcal{E}_{\mathrm{ext}}} \alpha_{\sigma} \tau_{\sigma} u_{K} v_{K}, \\
\ell_{\mathcal{D}}(v) & =\sum_{K \in \mathcal{T}} m(K) f_{K} v_{K}=\ell(v) .
\end{aligned}
$$


Using this notation, the flux formulation (3.1) is equivalent to searching for $u_{\gamma} \in H_{\mathcal{D}}(\Omega)$ such that we have the equality:

$$
a_{\gamma, \mathcal{D}}\left(u_{\gamma}, v\right)=\ell(v), \quad \forall v \in H_{\mathcal{D}}(\Omega) .
$$

We define a discrete $H_{0}^{1}(\Omega)$-like norm $\|\cdot\|_{1, \mathcal{D}}$ on $H_{\mathcal{D}}(\Omega)$ via

$$
\|u\|_{1, \mathcal{D}}^{2}=\sum_{K \mid L \in \mathcal{E}_{\mathrm{int}}} \tau_{K \mid L}\left(u_{L}-u_{K}\right)^{2}+\sum_{\sigma \in \mathcal{E}_{\mathrm{ext}}} \tau_{\sigma} u_{K}^{2} .
$$

\subsection{Strongly consistent discrete gradient}

In addition to (2.3), we need a good approximation of the gradient of piece-wise constant functions in order to evaluate the objective function (2.2) of (2.1). A construction of such an approximation is available, for example, in [22]. For an admissible finite volume discretization $\mathcal{D}$ of $\Omega$ the strongly consistent discrete gradient $\nabla_{\mathcal{D}}: H_{\mathcal{D}}(\Omega) \rightarrow\left[H_{\mathcal{D}}(\Omega)\right]^{d}$ is defined as follows: for $x \in K, K \in \mathcal{T}$ we set

$$
\nabla_{\mathcal{D}} u(x)=\frac{1}{m(K)}\left[\sum_{L \in \mathcal{N}_{K}} \tau_{K \mid L}\left(u_{L}-u_{K}\right)\left(x_{K \mid L}-x_{K}\right)-\sum_{\sigma \in \mathcal{E}_{K, \mathrm{ext}}} \tau_{\sigma} u_{K}\left(x_{K, \sigma}-x_{K}\right)\right] .
$$

The following is known about $\nabla_{\mathcal{D}}$ :

(i) For an admissible discretization $\mathcal{D}$ of $\Omega$ with $\theta_{\mathcal{D}}>0$, we have a uniform bound:

$$
\left\|\nabla_{\mathcal{D}} u\right\|_{\left[L^{2}(\Omega)\right]^{d}} \leq C\left(d, \theta_{\mathcal{D}}\right)\|u\|_{1, \mathcal{D}}, \quad \forall u \in H_{\mathcal{D}}(\Omega),
$$

where $C\left(d, \theta_{\mathcal{D}}\right)$ depends only on $d$ and $\theta_{\mathcal{D}}$; see [22], Lemma 2.2;

(ii) For an arbitrary sequence $\mathcal{D}^{(n)}$ of admissible discretizations of $\Omega$ such that $h_{\mathcal{D}^{(n)}} \rightarrow 0$ and $\theta_{\mathcal{D}} \geq \theta>0$, and for an arbitrary sequence $u^{(n)} \in H_{\mathcal{D}^{(n)}}$ such that $u^{(n)} \rightarrow \widehat{u} \in H_{0}^{1}(\Omega)$, strongly in $L^{2}(\Omega)$, it holds that $\nabla_{\mathcal{D}^{(n)}} u^{(n)} \rightarrow \nabla \widehat{u}$, weakly in $\left[L^{2}(\Omega)\right]^{d}[22]$, Lemma 2.3 .

(iii) For a sequence of discretizations as above and for an arbitrary $\phi \in C^{2}(\bar{\Omega})$ it holds that $\nabla_{\mathcal{D}^{(n)}} P_{\mathcal{D}^{(n)}} \phi \rightarrow$ $\nabla \phi$, strongly in $\left[L^{2}(\Omega)\right]^{d}[22]$, Lemma 2.5 .

\subsection{Cell-centered approximation of (2.1)}

Given an admissible finite volume discretization $\mathcal{D}$ of $\Omega$, and cell-centered approximation $G_{\mathcal{D}}$ of $G$, we will consider the following discretization of (2.1):

$$
\begin{array}{ll}
\text { minimize } & c_{\mathcal{D}}(\gamma), \\
\text { subject to } & \gamma \in G_{\mathcal{D}},
\end{array}
$$

where

$$
c_{\mathcal{D}}(\gamma)=\sum_{K \in \mathcal{T}} \int_{K} \mathcal{C}\left(x, \gamma_{K},\left[u_{\gamma}\right]_{K},\left[\nabla_{\mathcal{D}} u_{\gamma}\right]_{K}\right) \mathrm{d} x,
$$

and $u_{\gamma} \in H_{\mathcal{D}}(\Omega)$ is a solution to (3.5).

\section{Convergence of the scheme}

In this section we establish the convergence of optimal solutions to the cell-centered finite volume approximation scheme (3.8) towards optimal solutions of the control in the coefficients problem (2.1). The central result, Proposition 4.5, is an analogue of Proposition 2.2 for the cell-centered finite volume approximation (3.5) of BVP (2.6).

Unless some unrealistically restrictive assumptions are made about the design space $G$, we cannot count on any systematic limiting behaviour (as $h_{\mathcal{D}} \rightarrow 0$ ) of the traces $\alpha_{K \mid L}$ of the approximate diffusion coefficients on 
the boundaries of control volumes. Also, due to the nature of the cell-based finite volume approximation, both the diffusion coefficient and the solution to (3.5) have jumps across control volume boundaries. All this does not allow us to follow the convergence proof strategy utilized, e.g., in [22]: to show that the left hand side of (3.5) is close to $u_{K}[\operatorname{div}(\alpha \nabla \phi)]_{K}$ for every $\phi \in C_{c}^{\infty}(\Omega)$. In fact, the latter statement is not true any longer because the limiting coefficient $\alpha \notin C^{1}(\Omega)$. Instead, we use a technique inspired by the analysis of discontinuous Galerkin methods [14] and "lift" the approximations of boundary integrals involved in the variational formulation (3.5) back to the integrals over the control volumes. Namely, we show that the left hand side of (3.5) is close to $\alpha_{K}\left[\widehat{\nabla}_{\mathcal{D}} u\right]_{K} \cdot \nabla \phi\left(x_{K}\right)$ for every $\phi \in C_{c}^{\infty}(\Omega)$, where $\widehat{\nabla}_{\mathcal{D}} u$ is a weakly consistent approximation of the gradient of a piece-wise constant function. As it turns out, in the proof we cannot use the same consistent gradient approximation scheme utilized in [22], however a slight modification of this approximation (obtained by replacing $x_{K, \sigma}$ with $\left.z_{K, \sigma}\right)$ allows us to establish a convergence result.

\subsection{Existence of discrete solutions and uniform discrete compactness}

We start by recalling the existing results regarding discrete approximation problems (3.5) and (3.8).

Existence of discrete solutions to (3.5): For every admissible discretization $\mathcal{D}$ of $\Omega$ and every $\gamma \in G_{\mathcal{D}}$ we have the stability and continuity of the discrete bilinear form:

$$
\begin{array}{rlrl}
\|u\|_{1, \mathcal{D}}^{2} & \leq \underline{\alpha}^{-1} a_{\gamma, \mathcal{D}}(u, u), & & \forall u \in H_{\mathcal{D}}(\Omega), \\
a_{\gamma, \mathcal{D}}(u, v) & \leq \bar{\alpha}\|u\|_{1, \mathcal{D}}\|v\|_{1, \mathcal{D}}, \quad & \forall u, v \in H_{\mathcal{D}}(\Omega) .
\end{array}
$$

Therefore, Lax-Milgram theorem (see, for example, [3], Thm. 8.1) immediately implies that every discrete approximating problem (3.5) admits a unique solution $u_{\gamma} \in H_{\mathcal{D}}(\Omega)$.

Uniform boundedness: Further, for every $\gamma \in G$ we have a uniform (but mesh-dependent) estimate of the norm of solution $u_{\gamma} \in H_{\mathcal{D}}(\Omega)$ to $(3.5)$ :

$$
\underline{\alpha}\left\|u_{\gamma}\right\|_{1, \mathcal{D}}^{2} \leq a_{\gamma, \mathcal{D}}\left(u_{\gamma}, u_{\gamma}\right)=\ell\left(u_{\gamma}\right) \leq \underbrace{\left[\sup _{u \in H_{\mathcal{D}}(\Omega), u \neq 0} \frac{\ell(u)}{\|u\|_{1, \mathcal{D}}}\right]}_{=\|f\|_{-1, \mathcal{D}}}\left\|u_{\gamma}\right\|_{1, \mathcal{D}},
$$

where we utilized the definition of the norm $\|\cdot\|_{-1, \mathcal{D}}: L^{2}(\Omega) \rightarrow \mathbb{R}[21]$. Further, by the discrete Poincaré inequality [21], Lemma 9.1, we can majorize the latter mesh-dependent norm with a mesh-independent $L^{2}(\Omega)$ norm:

$$
\|f\|_{-1, \mathcal{D}} \leq \operatorname{diam}(\Omega)\|f\|_{L^{2}(\Omega)}
$$

Summarizing (4.1) and (4.2), for every $\gamma \in G_{\mathcal{D}}$, we have the following uniform estimate:

$$
\left\|u_{\gamma}\right\|_{1, \mathcal{D}} \leq \underline{\alpha}^{-1} \operatorname{diam}(\Omega)\|f\|_{L^{2}(\Omega)} .
$$

Existence of discrete solutions to (3.8): For every fixed discretization $\mathcal{D}$, the mapping $G_{\mathcal{D}} \ni \gamma \mapsto u_{\gamma} \in$ $H_{\mathcal{D}}(\Omega)$ is continuous owing to the implicit function theorem (e.g., [47], Thm. 4.E); hence so is the mapping $G_{\mathcal{D}} \ni \gamma \mapsto \nabla_{\mathcal{D}} u_{\gamma} \in\left[H_{\mathcal{D}}(\Omega)\right]^{d}$. Further, the set $G_{\mathcal{D}}$ is non-empty, closed and bounded, hence compact, and the cost function $G_{\mathcal{D}} \ni \gamma \mapsto c_{\mathcal{D}}(\gamma)=\int_{\Omega} \mathcal{C}\left(x, \gamma(x), u_{\gamma}(x), \nabla_{\mathcal{D}} u_{\gamma}\right) \mathrm{d} x$ is continuous. Therefore, by the virtue of Weierstrass' theorem (e.g., [8], Thm. 2.3.1) we obtain that every discrete approximating problem admits at least one globally optimal solution.

Discrete compactness: Now let $\mathcal{D}^{(n)}$ be a sequence of admissible discretizations of $\Omega$ such that $h_{\mathcal{D}^{(n)}} \rightarrow 0$ and $\gamma^{(n)} \in G_{\mathcal{D}^{(n)}}$ be arbitrary, and $u^{(n)} \in H_{\mathcal{D}^{(n)}}(\Omega)$ be the corresponding sequence of solutions to (3.5). Owing to the uniform estimate (4.3) and discrete Rellich-Kondrachov theorem [21], Theorem 14.2, the sequence $u^{(n)}$ is relatively compact in $L^{2}(\Omega)$ and we can extract a subsequence converging strongly in $L^{2}(\Omega)$ towards a limit $\widehat{u} \in H_{0}^{1}(\Omega)$. 


\subsection{Weakly consistent discrete gradient}

The following concept is what allows us to lift the edge-based variational form to a cell-based variational form in the proof of Lemma 4.3. For an admissible finite volume discretization $\mathcal{D}=(\mathcal{T}, \mathcal{E}, \mathcal{P})$ of $\Omega$ we define the weakly consistent discrete gradient $\widehat{\nabla}_{\mathcal{D}}: H_{\mathcal{D}}(\Omega) \rightarrow\left[H_{\mathcal{D}}(\Omega)\right]^{d}$ as follows: for $x \in K, K \in \mathcal{T}$ we set

$$
\widehat{\nabla}_{\mathcal{D}} u(x)=\frac{1}{m(K)}\left[\sum_{L \in \mathcal{N}_{K}} m(K \mid L)\left(u_{L}-u_{K}\right) \frac{d_{K, K \mid L}}{d_{K \mid L}} \mathbf{n}_{K \mid L}-\sum_{\sigma \in \mathcal{E}_{K, \text { ext }}} m(\sigma) u_{K} \mathbf{n}_{K, \sigma}\right] .
$$

The proofs in this subsection are almost verbatim repetitions of the ones found in [22] (as suggested by [22], Rem. 2.1) and are presented here only to keep the paper self-contained.

Lemma 4.1 (boundedness of $\widehat{\nabla}_{\mathcal{D}}$ ). For each $u \in H_{\mathcal{D}}(\Omega)$ we have the inequality:

$$
\left\|\widehat{\nabla}_{\mathcal{D}} u\right\|_{\left[L^{2}(\Omega)\right]^{d}}^{2} \leq 2 d\|u\|_{1, \mathcal{D}}^{2} .
$$

Proof. Fix an arbitrary $u \in H_{\mathcal{D}}$. We utilize Cauchy-Bunyakovsky-Schwarz inequality in (4.4) to write

$$
\begin{aligned}
m(K)^{2}\left|\left(\widehat{\nabla}_{\mathcal{D}} u\right)_{K}\right|^{2} & \leq\left[\sum_{L \in \mathcal{N}_{K}} m(K \mid L) \frac{d_{K, K \mid L}^{2}}{d_{K \mid L}}+\sum_{\sigma \in \mathcal{E}_{K, \mathrm{ext}}} m(\sigma) d_{K, \sigma}\right]\left[\sum_{L \in \mathcal{N}_{K}} \tau_{K \mid L}\left(u_{L}-u_{K}\right)^{2}+\sum_{\sigma \in \mathcal{E}_{K, \mathrm{ext}}} \tau_{\sigma} u_{K}^{2}\right] \\
& \leq d m(K)\left[\sum_{L \in \mathcal{N}_{K}} \tau_{K \mid L}\left(u_{L}-u_{K}\right)^{2}+\sum_{\sigma \in \mathcal{E}_{K, \mathrm{ext}}} \tau_{\sigma} u_{K}^{2}\right],
\end{aligned}
$$

where we used the facts that $d_{K, K \mid L} / d_{K \mid L} \leq 1, \sum_{\sigma \in \mathcal{E}_{K}} m(\sigma) d_{K, \sigma}=d m(K)$, and finally $\left|\mathbf{n}_{K, \sigma}\right|=1$. It remains to sum up the inequalities above over all finite volumes $K \in \mathcal{T}$ to obtain the desired estimate (4.5).

Lemma 4.2 (weak consistency of $\left.\widehat{\nabla}_{\mathcal{D}}\right)$. Let $\mathcal{D}^{(n)}=\left(\mathcal{T}^{(n)}, \mathcal{E}^{(n)}, \mathcal{P}^{(n)}\right)$ be a sequence of admissible discretizations of $\Omega$ such that $h_{\mathcal{D}^{(n)}} \rightarrow 0$ as $n \rightarrow \infty$. Further let $u^{(n)} \in H_{\mathcal{D}^{(n)}}(\Omega)$ be a sequence of functions converging to a limit $\widehat{u} \in H_{0}^{1}(\Omega)$, strongly in $L^{2}(\Omega)$, while remaining bounded: $\sup _{n}\left\|u^{(n)}\right\|_{1, \mathcal{D}^{(n)}}<\infty$. Then $\widehat{\nabla}_{\mathcal{D}^{(n)}} u^{(n)} \rightarrow \nabla \widehat{u}$, weakly in $\left[L^{2}(\Omega)\right]^{d}$.

Proof. Let us fix $\phi \in\left[C_{c}^{\infty}(\Omega)\right]^{d}$, and then choose a sufficiently large $n$ such that for every $K \in \mathcal{T}^{(n)}$ such that $K \cap \operatorname{supp}(\phi) \neq \emptyset$ it holds that $\partial K \cap \partial \Omega=\emptyset$.

We denote

and

$$
T_{1}^{(n)}=\int_{\Omega} \widehat{\nabla}_{\mathcal{D}^{(n)}} u^{(n)}(x) \cdot \phi(x) \mathrm{d} x
$$

$$
T_{2}^{(n)}=\int_{\Omega} \widehat{\nabla}_{\mathcal{D}^{(n)}} u^{(n)}(x) \cdot P_{\mathcal{D}^{(n)}} \phi(x) \mathrm{d} x .
$$

Owing to the boundedness of $\left\|u^{(n)}\right\|_{1, \mathcal{D}^{(n)}}$, Lemma 4.1, and the convergence $h_{\mathcal{D}^{(n)}} \rightarrow 0$ it holds that $\lim _{n \rightarrow \infty} \mid T_{1}^{(n)}$ $T_{2}^{(n)} \mid=0$. Using (4.4), we now rewrite $T_{2}^{(n)}$ as

$$
T_{2}^{(n)}=\sum_{K \mid L \in \mathcal{E}_{\text {int }}^{(n)}} m(K \mid L)\left(u_{L}^{(n)}-u_{K}^{(n)}\right) \frac{d_{K, K \mid L} \phi\left(x_{K}\right)+d_{L, K \mid L} \phi\left(x_{L}\right)}{d_{K \mid L}} \cdot \mathbf{n}_{K \mid L} .
$$

We compare $T_{2}^{(n)}$ with

$$
T_{3}^{(n)}=-\int_{\Omega} u^{(n)}(x) \operatorname{div} \phi(x) \mathrm{d} x=\sum_{K \mid L \in \mathcal{E}_{\text {int }}^{(n)}} m(K \mid L)\left(u_{L}^{(n)}-u_{K}^{(n)}\right) \frac{1}{m(K \mid L)} \int_{K \mid L} \phi(x) \cdot \mathbf{n}_{K \mid L} \mathrm{~d} x .
$$


Since $\phi \in\left[C_{c}^{\infty}(\Omega)\right]^{d}$ and $d_{K, K \mid L}+d_{L, K \mid L}=d_{K \mid L}$, it holds that

$$
\left|\frac{d_{K, K \mid L} \phi\left(x_{K}\right)+d_{L, K \mid L} \phi\left(x_{L}\right)}{d_{K \mid L}} \cdot \mathbf{n}_{K \mid L}-\frac{1}{m(K \mid L)} \int_{K \mid L} \phi(x) \cdot \mathbf{n}_{K \mid L} \mathrm{~d} x\right| \leq C_{\phi} h_{\mathcal{D}^{(n)}},
$$

where $C_{\phi}$ depends only on $\phi$. Therefore, by the virtue of Cauchy-Bunyakovsky-Schwarz inequality

$$
\begin{aligned}
\lim _{n \rightarrow \infty}\left(T_{2}^{(n)}-T_{3}^{(n)}\right)^{2} & \leq \lim _{n \rightarrow \infty}\left[C_{\phi}^{2} h_{\mathcal{D}^{(n)}}^{2} \sum_{K \mid L \in \mathcal{E}_{\text {int }}^{(n)}} \tau_{K \mid L}\left(u_{L}^{(n)}-u_{K}^{(n)}\right)^{2} \sum_{K \mid L \in \mathcal{E}_{\text {int }}^{(n)}} m(K \mid L) d_{K \mid L}\right] \\
& \leq C_{\phi}^{2} d m(\Omega)\left[\sup _{n}\left\|u^{(n)}\right\|_{1, \mathcal{D}^{(n)}}^{2}\right] \lim _{n \rightarrow \infty} h_{\mathcal{D}^{(n)}}^{2}=0 .
\end{aligned}
$$

Finally, owing to the strong convergence of $u^{(n)}$ to $\widehat{u}$ in $L^{2}(\Omega)$ we have

$$
\lim _{n \rightarrow \infty} T_{3}^{(n)}=-\int_{\Omega} \widehat{u}(x) \operatorname{div} \phi(x) \mathrm{d} x=\int_{\Omega} \nabla \widehat{u}(x) \cdot \phi(x) \mathrm{d} x .
$$

The proof is concluded owing to the density of $\left[C_{c}^{\infty}(\Omega)\right]^{d}$ in $\left[L^{2}(\Omega)\right]^{d}$.

\subsection{Convergence of the diffusion term}

Lemma 4.3. Let $\left(\mathcal{D}^{(n)}, \gamma^{(n)}, u^{(n)}\right)_{n \in \mathbb{N}}$ be a sequence of triples such that, for each $n \in \mathbb{N}, \mathcal{D}^{(n)}=\left(\mathcal{T}^{(n)}, \mathcal{E}^{(n)}, \mathcal{P}^{(n)}\right)$ is an admissible finite-volume discretization of $\Omega, \gamma^{(n)} \in G_{\mathcal{D}^{(n)}}$, and $u^{(n)} \in H_{\mathcal{D}^{(n)}}(\Omega)$. Assume further that $h_{\mathcal{D}^{(n)}} \rightarrow 0$ as $n \rightarrow \infty$, and $\gamma^{(n)} \rightarrow \widehat{\gamma} \in G$, a.e. in $\Omega$; as well as that $u^{(n)} \rightarrow \widehat{u} \in H_{0}^{1}(\Omega)$, strongly in $L^{2}(\Omega)$, while remaining bounded: $\sup _{n}\left\|u^{(n)}\right\|_{1, \mathcal{D}^{(n)}} \leq \widehat{C}<\infty$. Then for every $\phi \in C_{c}^{\infty}(\Omega)$ we have

$$
\lim _{n \rightarrow \infty} a_{\gamma^{(n)}, \mathcal{D}^{(n)}}\left(u^{(n)}, P_{\mathcal{D}^{(n)}} \phi\right)=a_{\widehat{\gamma}}(\widehat{u}, \phi) .
$$

Proof. Let us consider a sufficiently large $n$ such that for every $K \in \mathcal{T}^{(n)}$ such that $K \cap \operatorname{supp}(\phi) \neq \emptyset$ it holds that $\partial K \cap \partial \Omega=\emptyset$. We will write $\alpha^{(n)}=\alpha\left(\gamma^{(n)}\right), \widehat{\alpha}=\alpha(\widehat{\gamma})$; then $\alpha^{(n)} \rightarrow \widehat{\alpha}$, a.e. in $\Omega$. We set

$$
T_{1}^{(n)}=a_{\gamma^{(n)}, \mathcal{D}^{(n)}}\left(u^{(n)}, P_{\mathcal{D}^{(n)}} \phi\right)
$$

and

$$
T_{2}^{(n)}=\sum_{K \mid L \in \mathcal{E}_{\text {int }}^{(n)}} m(K \mid L)\left(u_{L}^{(n)}-u_{K}^{(n)}\right) \alpha_{K \mid L}^{(n)} \frac{1}{m(K \mid L)} \int_{K \mid L} \nabla \phi(x) \cdot \mathbf{n}_{K \mid L} \mathrm{~d} x .
$$

Then, the following chain of inequalities holds:

$$
\begin{aligned}
\lim _{n \rightarrow \infty}\left|T_{1}^{(n)}-T_{2}^{(n)}\right| & \leq \lim _{n \rightarrow \infty} \sum_{K \mid L \in \mathcal{E}_{\text {int }}^{(n)}} \tau_{K \mid L}\left|u_{L}^{(n)}-u_{K}^{(n)}\right| \alpha_{K \mid L}^{(n)} d_{K \mid L} \underbrace{\left|\frac{\phi\left(x_{L}\right)-\phi\left(x_{K}\right)}{d_{K \mid L}}-\frac{1}{m(K \mid L)} \int_{K \mid L} \nabla \phi(x) \cdot \mathbf{n}_{K \mid L} \mathrm{~d} x\right|}_{\leq C_{\phi} h_{\mathcal{D}^{(n)}}, \text { see }[21]} \\
& \leq \bar{\alpha} \lim _{n \rightarrow \infty}\left\|u^{(n)}\right\|_{1, \mathcal{D}^{(n)}}\left[\sum_{K \mid L \in \mathcal{E}_{\text {int }}^{(n)}} m(K \mid L) d_{K \mid L}\left[C_{\phi} h_{\mathcal{D}^{(n)}}\right]^{2}\right]^{1 / 2} \\
& \leq \bar{\alpha} \widehat{C} d m(\Omega) C_{\phi} \lim _{n \rightarrow \infty} h_{\mathcal{D}^{(n)}}=0
\end{aligned}
$$


where we used the equality $d_{K \mid L}^{-1}\left(x_{L}-x_{K}\right)=\mathbf{n}_{K \mid L}$ and the inequality $\sum_{K \mid L \in \mathcal{E}_{\text {int }}^{(n)}} \tau_{K \mid L} d_{K \mid L}^{2}=\sum_{K \mid L \in \mathcal{E}_{\text {int }}^{(n)}} m(K \mid$ $L) d_{K \mid L} \leq d m(\Omega) ; C_{\phi}$ is a generic positive constant depending on $\phi$ only. We now rewrite $T_{2}^{(n)}$ :

$$
T_{2}^{(n)}=\sum_{K \in \mathcal{T}^{(n)}} \sum_{L \in \mathcal{N}_{K}} m(K \mid L)\left(u_{L}^{(n)}-u_{K}^{(n)}\right) \frac{d_{K, K \mid L}}{d_{K \mid L}} \cdot \alpha_{K \mid L}^{(n)} \frac{1}{m(K \mid L)} \int_{K \mid L} \nabla \phi(x) \cdot \mathbf{n}_{K \mid L} \mathrm{~d} x,
$$

and define $T_{3}^{(n)}$ :

$$
T_{3}^{(n)}=\sum_{K \in \mathcal{T}^{(n)}} m(K) \alpha_{K}^{(n)} \widehat{\nabla}_{\mathcal{D}^{(n)}} u^{(n)} \cdot \nabla \phi\left(x_{K}\right) .
$$

If we could show that $T_{2}^{(n)}-T_{3}^{(n)} \rightarrow 0$ as $n \rightarrow \infty$, then the claim follows easily; we write

$$
\lim _{n \rightarrow \infty} T_{3}^{(n)}=\lim _{n \rightarrow \infty} \underbrace{\int_{\Omega} \alpha^{(n)} \widehat{\nabla}_{\mathcal{D}^{(n)}} u^{(n)} \cdot \nabla \phi \mathrm{d} x}_{T_{3,1}^{(n)}}+\lim _{n \rightarrow \infty} \underbrace{\int_{\Omega} \alpha^{(n)} \widehat{\nabla}_{\mathcal{D}^{(n)}} u^{(n)} \cdot\left[\nabla \phi-P_{\mathcal{D}^{(n)}} \nabla \phi\right] \mathrm{d} x}_{T_{3,2}^{(n)}} .
$$

Then,

$$
\lim _{n \rightarrow \infty}\left|T_{3,2}^{(n)}\right| \leq \bar{\alpha} \sup _{n}\left\|\widehat{\nabla}_{\mathcal{D}^{(n)}} u^{(n)}\right\|_{\left[L^{2}(\Omega)\right]^{d}} \lim _{n \rightarrow \infty}\left\|\nabla \phi-P_{\mathcal{D}^{(n)}} \nabla \phi\right\|_{\left[L^{2}(\Omega)\right]^{d}}=0,
$$

owing to the boundedness of $\left\|\widehat{\nabla}_{\mathcal{D}^{(n)}} u^{(n)}\right\|_{\left[L^{2}(\Omega)\right]^{d}}$ established in Lemma 4.1, and the fact that $h_{\mathcal{D}^{(n)}} \rightarrow 0$. On the other hand, since $\alpha^{(n)} \rightarrow \widehat{\alpha}$, a.e. in $\Omega$ while remaining bounded, and $\phi \in C_{c}^{\infty}(\Omega)$, then $\lim _{n \rightarrow \infty} \| \alpha^{(n)} \nabla \phi-$ $\widehat{\alpha} \nabla \phi \|_{\left[L^{2}(\Omega)\right]^{d}}=0$ and therefore

$$
\lim _{n \rightarrow \infty} T_{3,1}^{(n)}=a_{\widehat{\gamma}}(\widehat{u}, \phi),
$$

owing to the weak convergence of the gradient $\widehat{\nabla}_{\mathcal{D}^{(n)}} u^{(n)} \rightarrow \nabla \widehat{u}$ established in Lemma 4.2 .

Therefore, to conclude the proof it remains to show that $T_{2}^{(n)}-T_{3}^{(n)} \rightarrow 0$. Indeed,

$$
\begin{aligned}
T_{2}^{(n)}-T_{3}^{(n)}= & \sum_{K \in \mathcal{T}^{(n)}} \sum_{L \in \mathcal{N}_{K}} m(K \mid L)\left(u_{L}^{(n)}-u_{K}^{(n)}\right) \frac{d_{K, K \mid L}}{d_{K \mid L}}\left\{\alpha_{K \mid L}^{(n)} \frac{1}{m(K \mid L)} \int_{K \mid L} \nabla \phi(x) \mathrm{d} x-\alpha_{K}^{(n)} \nabla \phi\left(x_{K}\right)\right\} \cdot \mathbf{n}_{K \mid L} \\
= & \sum_{K \mid L \in \mathcal{E}_{\text {int }}^{(n)}} m(K \mid L)\left(u_{L}^{(n)}-u_{K}^{(n)}\right)\left\{\alpha_{K \mid L}^{(n)} \frac{1}{m(K \mid L)} \int_{K \mid L} \nabla \phi(x) \mathrm{d} x\right. \\
& \left.-\left[\frac{d_{K, K \mid L}}{d_{K \mid L}} \alpha_{K}^{(n)} \nabla \phi\left(x_{K}\right)+\frac{d_{L, K \mid L}}{d_{K \mid L}} \alpha_{L}^{(n)} \nabla \phi\left(x_{L}\right)\right]\right\} \cdot \mathbf{n}_{K \mid L}
\end{aligned}
$$

where we used the fact that $d_{K, K \mid L}+d_{L, K \mid L}=d_{K \mid L}$. Since $\alpha_{K \mid L}^{(n)}=d_{K, K \mid L} / d_{K \mid L} \alpha_{K}^{(n)}+d_{K, K \mid L} / d_{K \mid L} \alpha_{L}^{(n)}$, the last term in the curly braces can be majorized by $\bar{\alpha} C_{\phi} h_{\mathcal{D}}$. From here we obtain $\lim _{n \rightarrow \infty} T_{2}^{(n)}-T_{3}^{(n)}=0$ exactly as in (4.7).

Lemma 4.4 (strong convergence of the gradient). In addition to the assumptions of Lemma 4.3, assume that $\theta_{\mathcal{D}^{(n)}}>\theta>0$ for some $\theta>0$, and that

$$
\lim _{n \rightarrow \infty} a_{\gamma^{(n)}, \mathcal{D}^{(n)}}\left(u^{(n)}, u^{(n)}\right)=a_{\widehat{\gamma}}(\widehat{u}, \widehat{u}) .
$$

Then, $\nabla_{\mathcal{D}^{(n)}} u^{(n)}$ converges to $\nabla \widehat{u}$, strongly in $\left[L^{2}(\Omega)\right]^{d}$. 
Proof. For every $\phi \in C_{c}^{\infty}(\Omega)$ we have the inequality

$\left\|\nabla_{\mathcal{D}^{(n)}} u^{(n)}-\nabla \widehat{u}\right\|_{\left[L^{2}(\Omega)\right]^{d}} \leq\left\|\nabla_{\mathcal{D}^{(n)}} u^{(n)}-\nabla_{\mathcal{D}^{(n)}} P_{\mathcal{D}^{(n)}} \phi\right\|_{\left[L^{2}(\Omega)\right]^{d}}+\left\|\nabla_{\mathcal{D}^{(n)}} P_{\mathcal{D}^{(n)}} \phi-\nabla \phi\right\|_{\left[L^{2}(\Omega)\right]^{d}}+\|\nabla \phi-\nabla \widehat{u}\|_{\left[L^{2}(\Omega)\right]^{d}}$.

The last term on the right hand side can be made arbitrarily small by an appropriate choice of $\phi$ owing to the density of $C_{c}^{\infty}(\Omega)$ in $H_{0}^{1}(\Omega)$; the second term converges to zero for an arbitrary $\phi \in C_{c}^{\infty}(\Omega)$ when $n \rightarrow \infty$ owing to [22], Lemma 2.5. Therefore, it remains to estimate the first term.

Owing to the boundedness of the discrete gradient (Lem. 2.2 in [22]) we can write

$$
\begin{aligned}
\left\|\nabla_{\mathcal{D}^{(n)}}\left(u^{(n)}-P_{\mathcal{D}^{(n)}} \phi\right)\right\|_{\left[L^{2}(\Omega)\right]^{d}}^{2} & \leq C\left\|u^{(n)}-P_{\mathcal{D}^{(n)}} \phi\right\|_{1, \mathcal{D}^{(n)}}^{2} \leq \frac{C}{\underline{\alpha}} a_{\gamma^{(n)}, \mathcal{D}^{(n)}}\left(u^{(n)}-P_{\mathcal{D}^{(n)}} \phi, u^{(n)}-P_{\mathcal{D}^{(n)}} \phi\right) \\
& =\frac{C}{\underline{\alpha}}\left\{a_{\gamma^{(n)}, \mathcal{D}^{(n)}}\left(u^{(n)}, u^{(n)}\right)+a_{\gamma^{(n)}, \mathcal{D}^{(n)}}\left(-2 u^{(n)}+P_{\mathcal{D}^{(n)}} \phi, P_{\mathcal{D}^{(n)}} \phi\right)\right\}
\end{aligned}
$$

The first term in the curly braces converges to $a_{\widehat{\gamma}}(\widehat{u}, \widehat{u})$ by our assumption. Since $P_{\mathcal{D}^{(n)}} \phi \rightarrow \phi$, strongly in $L^{2}(\Omega)$ and $\left\|P_{\mathcal{D}^{(n)}} \phi\right\|_{1, \mathcal{D}^{(n)}} \leq C_{\phi}$ as can be easily verified, we can apply Lemma 4.3 to conclude that the second term converges to $a_{\widehat{\gamma}}(-2 \widehat{u}+\phi, \phi)$. Therefore,

$$
\limsup _{n \rightarrow \infty}\left\|\nabla_{\mathcal{D}^{(n)}}\left(u^{(n)}-P_{\mathcal{D}^{(n)}} \phi\right)\right\|_{\left[L^{2}(\Omega)\right]^{d}}^{2} \leq \frac{C}{\underline{\alpha}} a_{\widehat{\gamma}}(\widehat{u}-\phi, \widehat{u}-\phi) \leq C \frac{\bar{\alpha}}{\underline{\alpha}}\|\nabla \widehat{u}-\nabla \phi\|_{\left[L^{2}(\Omega)\right]^{d}}^{2},
$$

concluding the proof.

\subsection{Convergence of the approximations to (2.6) and (2.1)}

After the preliminary work done in Sections 4.1 through 4.3 we are ready to precisely formulate and prove an FVM-analogue of Proposition 2.2.

Proposition 4.5. Let $\mathcal{D}^{(n)}=\left(\mathcal{T}^{(n)}, \mathcal{E}^{(n)}, \mathcal{P}^{(n)}\right)$ be a sequence of admissible discretizations of $\Omega$ such that $h_{\mathcal{D}^{(n)}} \rightarrow 0$ as $n \rightarrow \infty$ and $\theta_{\mathcal{D}^{(n)}} \geq \theta$ for some $\theta>0$ and all $n \in \mathbb{N}$. Further, let $\gamma^{(n)} \in G_{\mathcal{D}^{(n)}}$ be a sequence of functions converging to $\widehat{\gamma} \in G$, a.e. in $\Omega$. Finally, let $u^{(n)} \in H_{\mathcal{D}^{(n)}}(\Omega)$ be a sequence of the unique solutions to (3.5) with $\gamma=\gamma^{(n)}$, and $\widehat{u} \in H_{0}^{1}(\Omega)$ be the unique solution to (2.6) corresponding to $\gamma=\widehat{\gamma}$. Then, $\| u^{(n)}-$ $\widehat{u} \|_{L^{2}(\Omega)} \rightarrow 0$ and $\left\|\nabla_{\mathcal{D}^{(n)}} u^{(n)}-\nabla \widehat{u}\right\|_{\left[L^{2}(\Omega)\right]^{d}} \rightarrow 0$.

Proof. Existence of the discrete solutions, their uniform (with respect to $\gamma^{(n)} \in G_{\mathcal{D}^{(n)}}$ ) boundedness, and relative compactness in $L^{2}(\Omega)$ have been discussed in Section 4.1. Therefore, we can apply Lemma 4.3 to every converging subsequence $u^{\left(n^{\prime}\right)}$ of $u^{(n)}$ :

$$
a_{\widehat{\gamma}}\left(\lim _{n^{\prime} \rightarrow \infty} u^{\left(n^{\prime}\right)}, \phi\right)=\lim _{n^{\prime} \rightarrow \infty} a_{\gamma^{\left(n^{\prime}\right)}, \mathcal{D}^{\left(n^{\prime}\right)}}\left(u^{\left(n^{\prime}\right)}, P_{\mathcal{D}^{\left(n^{\prime}\right)}} \phi\right)=\lim _{n^{\prime} \rightarrow \infty} \ell\left(P_{\mathcal{D}^{\left(n^{\prime}\right)}} \phi\right)=\ell(\phi), \quad \forall \phi \in C_{c}^{\infty}(\Omega) .
$$

Owing to the density of $C_{c}^{\infty}(\Omega)$ in $H_{0}^{1}(\Omega)$ we infer that every limit point must be a solution to (2.6) corresponding to $\gamma=\widehat{\gamma}$. Since the latter problem possesses a unique solution, namely $\widehat{u} \in H_{0}^{1}(\Omega)$, the whole sequence $u^{(n)}$ must converge to $\widehat{u}$, strongly in $L^{2}(\Omega)$. Finally, from (3.5) we infer that

$$
\lim _{n \rightarrow \infty} a_{\gamma^{(n)}, \mathcal{D}^{(n)}}\left(u^{(n)}, u^{(n)}\right)=\lim _{n \rightarrow \infty} \ell\left(u^{(n)}\right)=\ell(\widehat{u})=a_{\widehat{\gamma}}(\widehat{u}, \widehat{u})
$$

warranting the application of Lemma 4.4 and thus concluding the proof.

Remark 4.6. We note that the same conclusion can be reached if, instead of keeping the right hand side of (3.5) fixed, we assume that there is a sequence $f^{(n)} \in L^{2}(\Omega)$ converging weakly to $f \in L^{2}(\Omega)$. This fact will be used in the proof of convergence of FVM approximations to the adjoint problem (2.12). 
Finally, given Proposition 4.5 it is now straightforward to establish convergence of approximations (3.8) to the original control in the coefficients problem (2.1) assuming that $G_{\mathcal{D}}$ is a good approximation to $G$.

Theorem 4.7. Let $\mathcal{D}^{(n)}=\left(\mathcal{T}^{(n)}, \mathcal{E}^{(n)}, \mathcal{P}^{(n)}\right)$ be a sequence of admissible discretizations of $\Omega$ such that $h_{\mathcal{D}^{(n)}} \rightarrow 0$ as $n \rightarrow \infty$ and $\theta_{\mathcal{D}^{(n)}} \geq \theta$ for some $\theta>0$ and all $n \in \mathbb{N}$. Assume that the set of admissible designs $G$ is sequentially compact w.r.t. a.e. convergence in $\Omega$, thus guaranteeing the existence of the globally optimal solutions to (2.1). Further, let the discretized design sets $G_{\mathcal{D}^{(n)}}$ be closed, non-empty, and converge to $G$ in the following sense:

(i) For an arbitrary sequence $\widetilde{\gamma}^{(n)} \in G_{\mathcal{D}^{(n)}}$, its limit points w.r.t. a.e. convergence in $\Omega$ belong to $G$;

(ii) For an arbitrary point $\widetilde{\gamma} \in G$, there is a sequence $\widetilde{\gamma}^{(n)} \in G_{\mathcal{D}^{(n)}}$ such that $\widetilde{\gamma}^{(n)} \rightarrow \widetilde{\gamma}$, a.e. in $\Omega$.

(Note, that this assumption is satisfied when, e.g., $G_{\mathcal{D}^{(n)}} \rightarrow G$ in Painlevé-Kuratowski sense with respect to the distance induced by $L^{1}(\Omega)$-norm, also often found in practical setups of problems of control in the coefficients). Finally, let $\gamma^{(n)} \in G_{\mathcal{D}^{(n)}}$ be a sequence of globally optimal solutions to (3.8). Then, every limit point of this sequence with respect to a.e. convergence in $\Omega$ is a globally optimal solution to (2.1).

Proof. Let $\gamma^{*} \in G$ be an optimal solution to (2.1), and let $u_{\gamma^{*}} \in H_{0}^{1}(\Omega)$ be the corresponding solution to (2.6). By the assumed approximation property of $G_{\mathcal{D}^{(n)}}$ we can choose a sequence $\widetilde{\gamma}^{(n)} \in G_{\mathcal{D}^{(n)}}$ such that $\widetilde{\gamma}^{(n)} \rightarrow \gamma^{*}$, a.e. in $\Omega$. Therefore, owing to Proposition 4.5 and the assumption (2.5), we obtain the following inequality:

$$
\begin{aligned}
c\left(\gamma^{*}\right) & =\int_{\Omega} \mathcal{C}\left(x, \gamma^{*}(x), u_{\gamma^{*}}(x), \nabla u_{\gamma^{*}}(x)\right) \mathrm{d} x=\lim _{n \rightarrow \infty} \int_{\Omega} \mathcal{C}\left(x, \widetilde{\gamma}^{(n)}(x), u_{\widetilde{\gamma}^{(n)}}(x), \nabla_{\mathcal{D}^{(n)}} u_{\widetilde{\gamma}^{(n)}}(x)\right) \mathrm{d} x \\
& =\lim _{n \rightarrow \infty} c_{\mathcal{D}^{(n)}}\left(\widetilde{\gamma}^{(n)}\right) \geq \limsup _{n \rightarrow \infty} c_{\mathcal{D}^{(n)}}\left(\gamma^{(n)}\right) .
\end{aligned}
$$

On the other hand, let $\widehat{\gamma} \in G$ be a limit of a subsequence $\gamma^{\left(n^{\prime}\right)}$ w.r.t. a.e. convergence in $\Omega$, and let $u_{\widehat{\gamma}} \in H_{0}^{1}(\Omega)$ be the corresponding solution to (2.6). Then, $\widehat{\gamma} \in G$ and utilizing Proposition 4.5 and the condition (2.5) once more we get the opposite estimate:

$$
\begin{aligned}
c\left(\gamma^{*}\right) \leq c(\widehat{\gamma}) & =\int_{\Omega} \mathcal{C}\left(x, \widehat{\gamma}(x), u_{\widehat{\gamma}}(x), \nabla u_{\widehat{\gamma}}(x)\right) \mathrm{d} x=\lim _{n^{\prime} \rightarrow \infty} \int_{\Omega} \mathcal{C}\left(x, \gamma^{\left(n^{\prime}\right)}(x), u_{\gamma^{\left(n^{\prime}\right)}}(x), \nabla_{\mathcal{D}^{\left(n^{\prime}\right)}} u_{\gamma^{\left(n^{\prime}\right)}}(x)\right) \mathrm{d} x \\
& =\lim _{n^{\prime} \rightarrow \infty} c_{\mathcal{D}^{\left(n^{\prime}\right)}}\left(\gamma^{\left(n^{\prime}\right)}(x)\right) \leq \limsup _{n \rightarrow \infty} c_{\mathcal{D}^{(n)}}\left(\gamma^{(n)}(x)\right) .
\end{aligned}
$$

Therefore, $c\left(\gamma^{*}\right)=c(\widehat{\gamma})$, thus concluding the proof.

\section{Approximating (2.14): Discrete vs. Continuous adjoint approaches}

As was mentioned in Section 2.3, in practice we can only look for points which are stationary for the problem (2.1). For example, assuming the setup of Section 2.3, it is natural to look for points verifying a discrete version of stationarity conditions (2.14). There are at least two distinct ways of carrying out such a task, resulting in different numerical algorithms.

The first approach, which we will refer to as "the discrete adjoint"-based, and which is by far the most popular in the structural optimization community almost exclusively utilizing conforming finite element discretizations as an underlying numerical method for discretizing the governing PDEs [10]. The method can be concisely described as "discretize and then optimize." Namely, after the discretization (3.8) of (2.1) is obtained, it is treated as a regular non-linear mathematical program. That is, instead of the "continuous" stationarity conditions (2.14) we require that the discrete solutions $\gamma \in G_{\mathcal{D}}$ satisfy the standard first order necessary (KarushKuhn-Tucker) optimality conditions for (3.8). This approach has an advantage that one may immediately utilize a variety of standard non-linear mathematical programming algorithms for finding stationary points of (3.8). As we will show under some natural assumptions the latter points can be taken as approximations to "continuous" stationary points satisfying (2.14). 
The second approach, which we will call "the continuous adjoint"-based (see, for example, [32] and references therein), was advocated in the context of topology optimization for fluid dynamics in [36]. The method entails independent discretizations of the governing BVP (2.3), its adjoint (2.12), and the optimality conditions (2.14). It can be thought of as "optimize and then discretize" approach, because continuous optimality conditions are first stated and then discretized only afterwards. Within the software systems supporting the evaluation and manipulation of cell-based fields, such as for example OpenFOAM [1], this approach is somewhat more straighforward to implement than its discrete adjoint-based counterpart. This is of course advantageous for complicated multi-physics partial differential equations, but is less relevant for our problem (2.3). However, we note that one may no longer safely rely upon state-of-the-art non-linear programming algorithms for solving the discretized optimization problem to this special type of stationarity, which is a major disadvantage of this approach.

We formulate all the convergence results under the assumption that for every discretization we are able to find a solution, which satisfies stationarity conditions exactly. However, the fact that solutions to two different at the discrete level stationarity systems have the same limit points as the mesh size converges to zero indicates that this requirement may not be an essential one.

The associated convergence analysis with respect to mesh refinement is somewhat more straightforward for the continuous adjoint method that for the discrete one. For this reason, we start our investigation with the continuous adjoint-based approximation of the optimality system (2.14).

\subsection{Continuous adjoint-based approach}

We start with a given admissible finite volume discretization $\mathcal{D}=(\mathcal{T}, \mathcal{E}, \mathcal{P})$ of $\Omega$. The cell-based approximation of the adjoint problem (2.12) can be derived in the same way as the approximation (3.5) of the direct problem (2.6). We simply state the result:

$$
a_{\gamma, \mathcal{D}}\left(v, \lambda_{\gamma}\right)=\ell_{\mathrm{adj}, \mathcal{D}, \gamma, u_{\gamma}}(v), \quad \forall v \in H_{\mathcal{D}}(\Omega),
$$

with $\ell_{\mathrm{adj}, \mathcal{D}, \gamma, u_{\gamma}} \in\left[H_{\mathcal{D}}(\Omega)\right]^{*}$ given by

$$
\ell_{\mathrm{adj}, \mathcal{D}, \gamma, u_{\gamma}}(v)=-\sum_{K \in \mathcal{T}}\left\{\frac{1}{m(K)} \int_{K} \frac{\partial \mathcal{C}}{\partial u}\left(x, \gamma_{K},\left[u_{\gamma}\right]_{K}\right) \mathrm{d} x\right\} v_{K} m(K) .
$$

Now it remains to write a straightforward cell-based approximation of $D c(\gamma ; \widetilde{\gamma}-\gamma)$, see $(2.11)$, as

$$
\begin{aligned}
D c_{\mathcal{D}}(\gamma ; \widetilde{\gamma}-\gamma) & =\sum_{K \in \mathcal{T}}\left\{\frac{1}{m(K)} \int_{K} \frac{\partial \mathcal{C}}{\partial \gamma}\left(x, \gamma_{K},\left[u_{\gamma}\right]_{K}\right) \mathrm{d} x\right\}\left(\widetilde{\gamma}_{K}-\gamma_{K}\right) m(K) \\
& +\sum_{K \in \mathcal{T}} \alpha^{\prime}\left(\gamma_{K}\right)\left(\widetilde{\gamma}_{K}-\gamma_{K}\right)\left[\nabla_{\mathcal{D}} u_{\gamma}\right]_{K}\left[\nabla_{\mathcal{D}} \lambda_{\gamma}\right]_{K} m(K)
\end{aligned}
$$

where $\widetilde{\gamma}, \gamma \in G_{\mathcal{D}}, u_{\gamma} \in H_{\mathcal{D}}(\Omega)$ is the solution of the FVM approximation (3.5) of the direct problem (2.6), and $\lambda_{\gamma} \in H_{\mathcal{D}}(\Omega)$ is the solution of the FVM approximation (5.1) of the adjoint problem (2.12). Finally, the discretized version of (2.14) is just

$$
D c_{\mathcal{D}}\left(\gamma^{*} ; \gamma-\gamma^{*}\right) \geq 0 \quad \forall \gamma \in G_{\mathcal{D}}
$$

Proposition 5.1. Let us make the same assumptions and use the same notation as in Proposition 4.5. Additionally, assume that the conditions (i) and (ii) described in Section 2.3 hold. Then, the discretized adjoint problem (5.1) corresponding to $\gamma=\gamma^{(n)}, u=u^{(n)}$, admits a unique solution $\lambda^{(n)} \in H_{\mathcal{D}^{(n)}}(\Omega)$. Further, let $\widehat{\lambda} \in H_{0}^{1}(\Omega)$ be the solution to $(2.12)$ corresponding to $\gamma=\widehat{\gamma}, u=\widehat{u}$. Then, $\left\|\lambda^{(n)}-\widehat{\lambda}\right\|_{L^{2}(\Omega)} \rightarrow 0$ and $\left\|\nabla_{\mathcal{D}^{(n)}} \lambda^{(n)}-\nabla \widehat{\lambda}\right\|_{\left[L^{2}(\Omega)\right]^{d}} \rightarrow 0$. 
Proof. The existence and the uniqueness of the adjoint solution $\lambda^{(n)} \in H_{\mathcal{D}^{(n)}}(\Omega)$ is a consequence of the LaxMilgram theorem, exactly as discussed in Subsection 4.1. Owing to Proposition 4.5 and continuity (2.10) we have the convergence $\partial \mathcal{C} / \partial u\left(\cdot, \gamma^{(n)}(\cdot), u^{(n)}(\cdot)\right) \rightarrow \partial \mathcal{C} / \partial u(\cdot, \widehat{\gamma}(\cdot), \widehat{u}(\cdot))$, weakly in $L^{2}(\Omega)$. As mentioned in Remark 4.6, we can now repeat the arguments of Proposition 4.5 verbatim for the adjoint problem (5.1) to conclude that $\left\|\lambda^{(n)}-\widehat{\lambda}\right\|_{L^{2}(\Omega)} \rightarrow 0$ and $\left\|\nabla_{\mathcal{D}^{(n)}} \lambda^{(n)}-\nabla \widehat{\lambda}\right\|_{\left[L^{2}(\Omega)\right]^{d}} \rightarrow 0$, as claimed.

Since the variational inequality (5.4) is a discretization of the continuous optimality conditions (2.14) and does not describe a set of optimality conditions for a discrete optimization problem, we have to appeal directly to the theory of (discrete) variational inequalities to establish the existence of stationary points in the sense of (5.4).

Lemma 5.2. Let $G_{\mathcal{D}}$ be non-empty, closed, bounded and convex. Then, the variational inequality problem (5.4) admits at least one solution $\gamma^{*} \in G_{\mathcal{D}}$.

Proof. The mapping $G_{\mathcal{D}} \ni \gamma \mapsto\left\{\alpha^{\prime}\left(\gamma_{K}\right)\left[\nabla_{\mathcal{D}} u_{\gamma}\right]_{K}\left[\nabla_{\mathcal{D}} \lambda_{\gamma}\right]_{K} m(K)\right\}_{K \in \mathcal{T}} \in \mathbb{R}^{|\mathcal{T}|}$ is continuous as a composition of the continuous mappings $G_{\mathcal{D}} \ni \gamma \mapsto u_{\gamma} \in H_{\mathcal{D}}(\Omega), G_{\mathcal{D}} \times H_{\mathcal{D}}(\Omega) \ni\left(\gamma, u_{\gamma}\right) \mapsto \lambda_{\gamma} \in H_{\mathcal{D}}(\Omega),\left[H_{\mathcal{D}}(\Omega)\right]^{2} \ni$ $\left(u_{\gamma}, \lambda_{\gamma}\right) \mapsto\left(\nabla_{\mathcal{D}} u_{\gamma}, \nabla_{\mathcal{D}} \lambda_{\gamma}\right) \in\left[H_{\mathcal{D}}(\Omega)\right]^{2 d}$, and $[0,1] \ni \gamma \mapsto \alpha^{\prime}(\gamma)$. Furthermore, the set $G_{\mathcal{D}}$ is non-empty, convex, and compact. Under this condition, the variational inequality problem (5.4) is known to admit solutions, see for example [38], Theorem 4.1, for details.

Now that we know the discrete stationary points (5.4) exist, we can establish a practical analogue of Theorem 4.7 asserting that stationary points (5.4) can only converge towards stationary points (2.14).

Theorem 5.3. Let $\mathcal{D}^{(n)}=\left(\mathcal{T}^{(n)}, \mathcal{E}^{(n)}, \mathcal{P}^{(n)}\right)$ be a sequence of admissible discretizations of $\Omega$ such that $h_{\mathcal{D}^{(n)}} \rightarrow 0$ as $n \rightarrow \infty$ and $\theta_{\mathcal{D}^{(n)}} \geq \theta$ for some $\theta>0$ and all $n \in \mathbb{N}$. Assume that the set of admissible designs $G$ is closed w.r.t. a.e. convergence on $\Omega$ and convex. Further, let the discretized design sets $G_{\mathcal{D}^{(n)}}$ be closed, convex, non-empty, and converge to $G$ in the same way as in Theorem 4.\%. Finally, let $\gamma^{(n)} \in G_{\mathcal{D}^{(n)}}$ be a sequence of stationary points, that is, solutions to (5.4). Then, every limit point of this sequence with respect to a.e. convergence on $\Omega$ is stationary for (2.1), that is, it verifies (2.14).

Proof. Let $\widehat{\gamma}=$ a.e.- $\lim _{n^{\prime} \rightarrow \infty} \gamma^{\left(n^{\prime}\right)}$, for some subsequence of indices $n^{\prime}$, and let us fix an arbitrary $\widetilde{\gamma} \in G$. By the assumptions of the theorem, $\widehat{\gamma} \in G$ and there is a sequence $\widetilde{\gamma}^{(n)} \in G_{\mathcal{D}^{(n)}}$ such that $\widetilde{\gamma}=$ a.e.- $\lim _{n \rightarrow \infty} \widetilde{\gamma}^{(n)}$. Owing to Propositions 4.5 and 5.1, as well as the condition (2.10) for $\partial \mathcal{C} / \partial \gamma, \partial \mathcal{C} / \partial u$, we can infer that

$$
D c(\widehat{\gamma} ; \widetilde{\gamma}-\widehat{\gamma})=\lim _{n^{\prime} \rightarrow \infty} D c_{\mathcal{D}^{\left(n^{\prime}\right)}}\left(\gamma^{\left(n^{\prime}\right)} ; \widetilde{\gamma}^{\left(n^{\prime}\right)}-\gamma^{\left(n^{\prime}\right)}\right) \geq 0
$$

where the last inequality is due to the stationarity of $\gamma^{\left(n^{\prime}\right)}$, that is, (5.4).

\subsection{Discrete adjoint-based approach}

Now we look at the discrete adjoint-based approach to optimality conditions. Utilizing the implicit function theorem and the chain rule, we compute the partial derivatives of $c_{\mathcal{D}}$ with respect to $\gamma$ as:

$$
\begin{aligned}
\frac{\partial c_{\mathcal{D}}}{\partial \gamma_{K}}(\gamma)= & \int_{K} \frac{\partial \mathcal{C}}{\partial \gamma}\left(x, \gamma_{K},\left[u_{\gamma}\right]_{K}\right) \mathrm{d} x \\
& +\sum_{L \in \mathcal{N}_{K}} \alpha^{\prime}\left(\gamma_{K}\right) \frac{d_{K, K \mid L}}{d_{K \mid L}} \tau_{K \mid L}\left(\left[u_{\gamma}\right]_{L}-\left[u_{\gamma}\right]_{K}\right)\left(\left[\lambda_{\gamma}\right]_{L}-\left[\lambda_{\gamma}\right]_{K}\right) \\
& +\sum_{\sigma \in \mathcal{E}_{K, \text { ext }}} \alpha^{\prime}\left(\gamma_{K}\right) \tau_{\sigma}\left[u_{\gamma}\right]_{K}\left[\lambda_{\gamma}\right]_{K}
\end{aligned}
$$


where, as before, $u_{\gamma} \in H_{\mathcal{D}}(\Omega)$ is a solution to (3.5) and $\lambda_{\gamma} \in H_{\mathcal{D}}(\Omega)$ is a solution to (5.1). Therefore, the directional derivative, $\widehat{D} c_{\mathcal{D}}(\gamma ; \widetilde{\gamma}-\gamma)=\left[\nabla_{\gamma} c_{\mathcal{D}}(\gamma)\right]^{\mathrm{T}}(\widetilde{\gamma}-\gamma)$, can be written as

$$
\begin{aligned}
\widehat{D} c_{\mathcal{D}}(\gamma ; \widetilde{\gamma}-\gamma)= & \sum_{K \in \mathcal{T}}\left\{\frac{1}{m(K)} \int_{K} \frac{\partial \mathcal{C}}{\partial \gamma}\left(x, \gamma_{K},\left[u_{\gamma}\right]_{K}\right) \mathrm{d} x\right\}\left(\widetilde{\gamma}_{K}-\gamma_{K}\right) m(K) \\
& +\sum_{K \mid L \in \mathcal{E}_{\mathrm{int}}} \delta_{K \mid L} \tau_{K \mid L}\left(\left[u_{\gamma}\right]_{L}-\left[u_{\gamma}\right]_{K}\right)\left(\left[\lambda_{\gamma}\right]_{L}-\left[\lambda_{\gamma}\right]_{K}\right) \\
& +\sum_{\sigma \in \mathcal{E}_{K, \mathrm{ext}}} \delta_{K} \tau_{\sigma}\left[u_{\gamma}\right]_{K}\left[\lambda_{\gamma}\right]_{K},
\end{aligned}
$$

where we introduced a shorthand notation $\delta_{K}=\alpha^{\prime}\left(\gamma_{K}\right)\left(\widetilde{\gamma_{K}}-\gamma_{K}\right)$ and $\delta_{K \mid L}=\alpha^{\prime}\left(\gamma_{K}\right)\left(\widetilde{\gamma}_{K}-\gamma_{K}\right) d_{K, K \mid L} / d_{K \mid L}+$ $\alpha^{\prime}\left(\gamma_{L}\right)\left(\widetilde{\gamma}_{L}-\gamma_{L}\right) d_{L, K \mid L} / d_{K \mid L}$, which is consistent with (3.3).

If $\gamma^{*} \in G_{\mathcal{D}}$ is a local minimum in (3.8), then the following variational inequality holds (e.g., [11], Lem. 3.7):

$$
\widehat{D} c_{\mathcal{D}}\left(\gamma^{*} ; \gamma-\gamma^{*}\right) \geq 0 \quad \forall \gamma \in G_{\mathcal{D}}
$$

which is just a set of first order necessary optimality conditions for (3.8) taking into account the convexity of $G_{\mathcal{D}}$. Assuming compactness of the approximating sets $G_{\mathcal{D}}$, the variational inequality problem $(5.7)$ has at least one solution by the virtue of Weierstrass' theorem (e.g., [8], Thm. 2.3.1) and the necessity of (5.7) for optimality. Therefore, we can directly proceed to identifying the limit of $\widehat{D} c_{\mathcal{D}}$ when $h_{\mathcal{D}} \rightarrow 0$.

Lemma 5.4. Let us make the same assumptions and use the same notation as in Proposition 5.1. Additionally, assume that a sequence $\widetilde{\gamma}^{(n)} \in G_{\mathcal{D}^{(n)}}$ converges, a.e. in $\Omega$, towards a limit $\widetilde{\gamma} \in G$. Then, $\lim _{n \rightarrow \infty} \widehat{D} c_{\mathcal{D}^{(n)}}\left(\gamma^{(n)} ; \widetilde{\gamma}^{(n)}-\right.$ $\left.\gamma^{(n)}\right)=D c(\widehat{\gamma} ; \widetilde{\gamma}-\widehat{\gamma})$.

Proof. Let us analyze the terms involved in $\widehat{D} c_{\mathcal{D}^{(n)}}\left(\gamma^{(n)} ; \widetilde{\gamma}^{(n)}-\gamma^{(n)}\right)$, see (5.7), individually. The sum over control volumes converges towards $\int_{\Omega} \partial \mathcal{C} / \partial \gamma(x, \widehat{\gamma}(x), \widehat{u}(x))(\widetilde{\gamma}(x)-\widehat{\gamma}(x)) \mathrm{d} x$, owing to Proposition 4.5 and condition (2.10), exactly as in Theorem 5.3.

To analyze the edge-wise sums we proceed as follows. Let us fix an arbitrary $\phi \in C_{c}^{\infty}(\Omega)$ and then choose a sufficiently large $n$ such that for every $K \in \mathcal{T}^{(n)}$ such that $K \cap \operatorname{supp}(\phi) \neq \emptyset$ it holds that $\partial K \cap \partial \Omega=\emptyset$. Then,

$$
\begin{aligned}
& \sum_{K \mid L \in \mathcal{E}_{\mathrm{int}}^{(n)}} \delta_{K \mid L}^{(n)} \tau_{K \mid L}\left(u_{L}^{(n)}-u_{K}^{(n)}\right)\left(\lambda_{L}^{(n)}-\lambda_{K}^{(n)}\right)+\sum_{\sigma \in \mathcal{E}_{K, \text { ext }}^{(n)}} \delta_{K}^{(n)} \tau_{\sigma} u_{K}^{(n)} \lambda_{K}^{(n)} \\
= & \sum_{T_{1}^{(n)}}^{\sum_{K \mid L \in \mathcal{E}_{\mathrm{int}}^{(n)}} \delta_{K \mid L}^{(n)} \tau_{K \mid L}\left(u_{L}^{(n)}-u_{K}^{(n)}\right)\left(\phi\left(x_{L}\right)-\phi\left(x_{K}\right)\right)} \\
+ & \underbrace{\sum_{K \mid L \in \mathcal{E}_{\mathrm{int}}^{(n)}} \delta_{K \mid L}^{(n)} \tau_{K \mid L}\left(u_{L}^{(n)}-u_{K}^{(n)}\right)\left(\left[\lambda_{L}^{(n)}-\phi\left(x_{L}\right)\right]-\left[\lambda_{K}^{(n)}-\phi\left(x_{K}\right)\right]\right)+\sum_{\sigma \in \mathcal{E}_{K, \mathrm{ext}}^{(n)}} \delta_{K}^{(n)} \tau_{\sigma} u_{K}^{(n)}\left[\lambda_{K}^{(n)}-\phi\left(x_{K}\right)\right]}_{T_{2}^{(n)}}
\end{aligned}
$$

where we used the notation $\delta_{K}^{(n)}=\alpha^{\prime}\left(\gamma_{K}^{(n)}\right)\left(\widetilde{\gamma}_{K}^{(n)}-\gamma_{K}^{(n)}\right)$, with $\delta_{K \mid L}^{(n)}$ defined by weighted arithmetic averaging exactly as in (3.3). The term $T_{1}^{(n)}$ can be easily seen to converge to $\int_{\Omega} \alpha^{\prime}(\widehat{\gamma}(x))(\widetilde{\gamma}(x)-\widehat{\gamma}(x)) \nabla \widehat{u}(x) \cdot \nabla \phi(x) \mathrm{d} x$, by repeating the arguments of Lemma 4.3 while substituting $\alpha\left(\gamma^{(n)}\right)$ with $\alpha^{\prime}\left(\gamma^{(n)}\right)\left(\widetilde{\gamma}^{(n)}-\gamma^{(n)}\right)$. On the other 
hand, owing to Cauchy-Bunyakovsky-Schwarz inequality, we get the estimate

$$
\begin{aligned}
\limsup _{n \rightarrow \infty}\left|T_{2}^{(n)}\right| & \leq 2\left[\sup _{y \in[0,1]}\left|\alpha^{\prime}(y)\right|\right]\left[\sup _{n \in \mathbb{N}}\left\|u^{(n)}\right\|_{1, \mathcal{D}^{(n)}}\right] \limsup _{n \rightarrow \infty}\left\|\lambda^{(n)}-P_{\mathcal{D}^{(n)}} \phi\right\|_{1, \mathcal{D}^{(n)}} \\
& \leq 2\left[\sup _{y \in[0,1]}\left|\alpha^{\prime}(y)\right|\right]\left[\sup _{n \in \mathbb{N}}\left\|u^{(n)}\right\|_{1, \mathcal{D}^{(n)}}\right] \bar{\alpha}^{1 / 2} \underline{\alpha}^{-1 / 2}\|\nabla \hat{\lambda}-\nabla \phi\|_{L^{2}(\Omega)},
\end{aligned}
$$

where the last inequality can be obtained by utilizing the same "trick" as in (4.11). Therefore, we can conclude the proof by letting $\phi \rightarrow \widehat{\lambda}$ in $H_{0}^{1}(\Omega)$.

After this preliminary work, we can formulate a direct analogue of Theorem 5.3 for the discrete adjoint-based approach to stationarity.

Theorem 5.5. Let $\mathcal{D}^{(n)}=\left(\mathcal{T}^{(n)}, \mathcal{E}^{(n)}, \mathcal{P}^{(n)}\right)$ be a sequence of admissible discretizations of $\Omega$ such that $h_{\mathcal{D}^{(n)}} \rightarrow 0$ as $n \rightarrow \infty$ and $\theta_{\mathcal{D}^{(n)}} \geq \theta$ for some $\theta>0$ and all $n \in \mathbb{N}$. Assume that the set of admissible designs $G$ is closed w.r.t. a.e. convergence on $\Omega$ and convex. Further, let the discretized design sets $G_{\mathcal{D}^{(n)}}$ be closed, convex, nonempty, and converge to $G$ in the same way as in Theorem 4.7. Finally, let $\gamma^{(n)} \in G_{\mathcal{D}^{(n)}}$ be a sequence of KKT points, that is, solutions to (5.7). Then, every limit point of this sequence with respect to a.e. convergence on $\Omega$ is stationary for (2.1), that is, it verifies (2.14).

Proof. Repeat the arguments of the proof of Theorem 5.3 while substituting $D c_{\mathcal{D}^{(n)}}$ with $\widehat{D} c_{\mathcal{D}^{(n)}}$ and utilizing Lemma 5.4 when obtaining the analogue of (5.5).

\section{NUMERICAL EXPERIMENT}

To illustrate the practical behaviour of a cell-based FVM topology optimization method we apply it on an instance of a problem of control in the conduction coefficients. Physically, we model a problem with a "hot spot" at $x^{0}=(0.501,0.7,-0.5) \in \mathbb{R}^{3}$ and a "cold spot" at $x^{1}=(0.499,0.3,-0.5) \in \mathbb{R}^{3}$ in the plane $\left\{x=\left(x_{1}, x_{2}, x_{3}\right) \in \mathbb{R}^{3} \mid x_{3}=-0.5\right\}$. By distributing a high conductive material and a low conductive material we try to "change the sign" of these spots in the plane $\left\{\left(x_{1}, x_{2}, x_{3}\right) \in \mathbb{R}^{3} \mid x_{3}=0.0\right\}$, that is we would like to have a cold spot at $\hat{x}^{0}=(0.5,0.7,0.0) \in \mathbb{R}^{3}$ and a hot spot at $\hat{x}^{1}=(0.5,0.3,0.0) \in \mathbb{R}^{3}$.

Mathematically, we formulte such a problem as follows. The computational domain $\Omega$ is set to $(0.0,1.0)^{2} \times$ $(-0.5,0.0) \subset \mathbb{R}^{3}$. We prescribe homogeneous Neumann boundary conditions on all faces of the polygon except on the plane $\left\{\left(x_{1}, x_{2}, x_{3}\right) \in \mathbb{R}^{3} \mid x_{3}=-0.5\right\}$ we prescribe Dirichlet boundary conditions $u=100\{\exp [-100 \| x-$ $\left.\left.x^{0} \|_{2}^{2}\right]-\exp \left[-100\left\|x-x^{1}\right\|_{2}^{2}\right]\right\}$. In the adjoint problem these conditions correspond to homogeneous Neumann boundary conditions on all faces except on the plane $\left\{\left(x_{1}, x_{2}, x_{3}\right) \in \mathbb{R}^{3} \mid x_{3}=-0.5\right\}$ one demands homogeneous Dirichlet boundary conditions. We set $G=\left\{\gamma \in L^{\infty}(\Omega) \mid \gamma(x) \in[0,1]\right.$, a.e. in $\left.\Omega\right\}, \underline{\alpha}=0.01$ and $\bar{\alpha}=1$. Finally, $\mathcal{C}(x, \gamma, u)=100\left\{\exp \left[-100\left\|x-\hat{x}^{0}\right\|_{2}^{2}\right]-\exp \left[-100\left\|x-\hat{x}^{1}\right\|_{2}^{2}\right]\right\} u$, and $\alpha(\gamma)=\underline{\alpha}+(\bar{\alpha}-\underline{\alpha}) \gamma /[1.0+6.0(1.0-\gamma)]$.

We discretize the problem with a regular grid of $150 \times 150 \times 75 \approx 1.7 \cdot 10^{6}$ cells and approximately $5.1 \times 10^{6}$ faces. We implement our algorithm within the open source finite volume method framework OpenFOAM [1]. We use a separable convex approximation algorithm MMA [43,44], favoured by the structural optimization community, which we supply with the first order information obtained from either (5.3) or (5.7). We start the algorithm from the initial design $\gamma_{0} \equiv 0.5$, and we stop it either when the KKT residual drops below $1.0 \times 10^{-5}$ or when the maximum number of iterations, set to 200 , is reached.

The results of this numerical experiment are summarized in Table 1 and Figure 3. We can note that the optimization algorithm on this problem instance found two very similar solutions, which differ in terms of the objective function by less than $1 \%$. We also note that in the case of inaccurate gradient information (5.3) provided by the continuous adjoint approach the algorithm fails to reduce the KKT residual below the preset threshold, which is to be expected. A curious incident is that, on this problem instance, based on such inaccurate information the optimization algorithm finds a slightly better solution in terms of the objective function. 


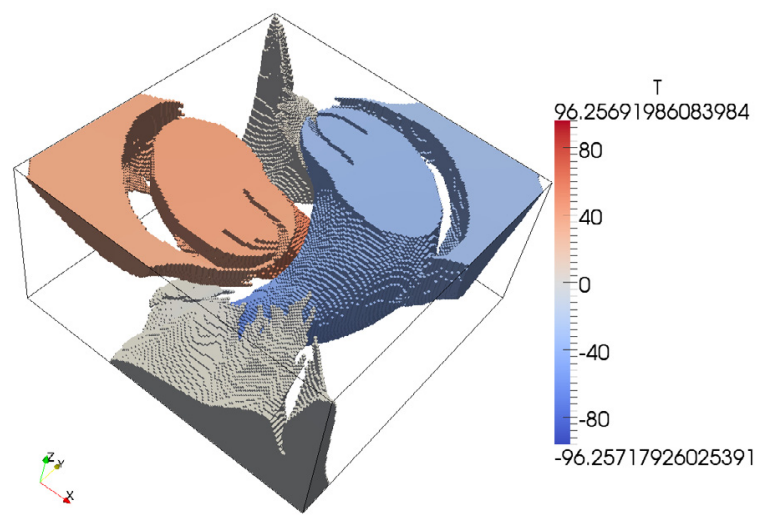

(a)

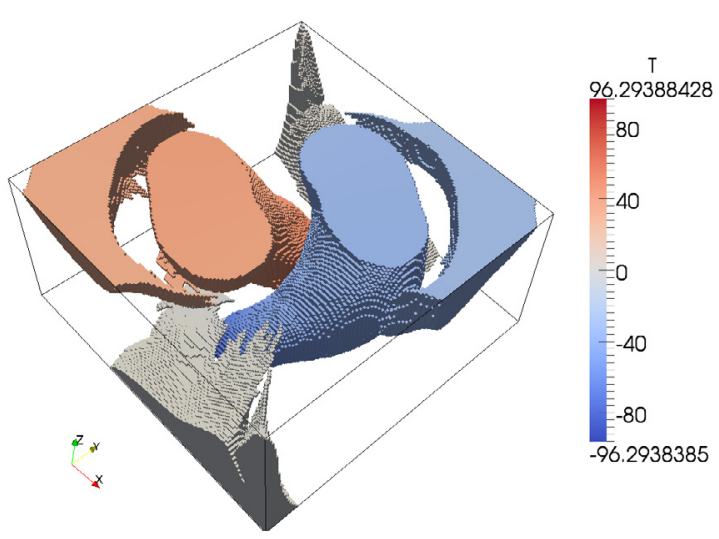

(b)

Figure 3. Optimal designs obtained in the numerical experiment using (a) continuous adjoint approach; and (b) discrete adjoint approach. We only display finite folume cells corresponding to $\gamma \geq 0.5$ and colour them with values of the "temperature" $T$.

TABLE 1. Summary of the quantitative data obtained in the numerical experiment. \# iter: number of optimization iterations; Obj. f-n: objective function value; KKT: a measure of the KKT residual at the termination point; " $0-1$ ": a value of the integral $(4 m(\Omega))^{-1} \int_{\Omega} \gamma(x)[1.0-$ $\gamma(x)] \mathrm{d} x$ measuring the deviation of values of $\gamma$ from extremal values 0 and 1 .

\begin{tabular}{l|cccc}
\hline & \# iter & Obj. f-n & KKT & "0-1" \\
\hline Continuous adjoint & 200 & -19.431 & $6.329 \times 10^{-5}$ & $1.122 \times 10^{-3}$ \\
Discrete adjoint & 129 & -19.278 & $8.965 \times 10^{-6}$ & $1.914 \times 10^{-3}$ \\
\hline
\end{tabular}

\section{Conclusions And Future RESEArch}

Despite the difficulties outlined in the introduction and contrary to our intuition, we have been able to establish continuity of the coefficient-to-solution mapping resulting from the finite volume discretizations of problems of control in the coefficients of generalized Laplace equation without assuming any additional regularity of the solutions and without unnecessarily restrictive assumptions on the convergence of the coefficients. As a result, we have been able to characterize limit points of the sequences of discrete global solutions and two types of stationary points, as the finite volume mesh size converges to zero.

More numerical testing of the algorithm resulting from FVM discretization of control in the coefficients problems has to be carried out; however, in this study we concentrate on the convergence analysis of the algorithm.

Finally, we note that this study is a first step in the direction of enabling finite volume discretizations of control in the coefficient problems. Of great practical importance is relaxing or removing the mesh orthogonality requirement assumed throughout this paper, as well as applying the method to problems involving several physical phenomena, such as for example coupled mass and heat transfer.

\section{REFERENCES}

[1] http://www.openfoam.com.

[2] N. Aage, T.H. Poulsen, A. Gersborg-Hansen and O. Sigmund, Topology optimization of large scale Stokes flow problems. Struct. Multidisc. Optim. 35 (2008) 175-180.

[3] S. Agmon, Lectures on elliptic boundary value problems. Van Nostrand, Princeton, N.J. (1965). 
[4] G. Allaire, Conception optimale de structures, Mathématiques et Applications 58. Springer (2007).

[5] L. Ambrosio and G. Buttazzo, An optimal design problem with perimeter penalization. Calc. Var. Partial Differential Equations 1 (1993) 55-69.

[6] C.S. Andreasen, A.R. Gersborg and Ole Sigmund, Topology optimization of microfluidic mixers. Int. J. Numer. Methods Fluids 61 (2008) 498-513.

[7] H. Attouch, G. Buttazzo and G. Michaille, Variational analysis in Sobolev and BV spaces: applications to PDEs and optimization. SIAM (2006) 648. ISBN 9780898716009.

[8] M.S. Bazaraa, H.D. Sherali and C.M. Shetty, Nonlinear Programming. John Wiley \& Sons, Inc, New York (1993).

[9] M.P. Bendsøe and N. Kikuchi, Generating optimal topologies in structural design using a homogenization method. Comput. Methods Appl. Mech. Engrg. 71 (1988) 197-224. CODEN CMMECC. ISSN 0045-7825.

[10] M.P. Bendsøe and O. Sigmund, Topology Optimization: Theory, Methods, and Applications. Springer-Verlag, Berlin (2003). 370. ISBN 3-540-42992-1.

[11] J.F. Bonnans and A. Shapiro, Perturbation Analysis of Optimization Problems. Springer-Verlag, New York (2000), p. 601. ISBN 0-387-98705-3.

[12] T. Borrvall and J. Petersson, Topology optimization of fluids in Stokes flow. Int. J. Numer. Methods Fluids 41 (2003) $77-107$. CODEN IJNFDW. ISSN 0271-2091.

[13] B. Dacorogna, Direct methods in the calculus of variations, Applied Mathematical Sciences 78. Springer-Verlag, Berlin (1989). $\mathrm{x}+308$ ISBN 3-540-50491-5.

[14] D.A. Di Pietro and A. Ern, Discrete functional analysis tools for Discontinuous Galerkin methods with application to the incompressible Navier-Stokes equations. Math. Comput. 79 (2010) 1303-1330.

[15] L.C. Evans and R.F. Gariepy, Measure theory and fine properties of functions. CRC Press (1992).

[16] A. Evgrafov, On the limits of porous materials in the topology optimization of Stokes flows. Appl. Math. Optim. 52 (2005) 263-267.

[17] A. Evgrafov, Topology optimization of slightly compressible fluids. Z. Angew. Math. Mech. 86 (2005) 46-62.

[18] A. Evgrafov, G. Pingen and K. Maute, Topology optimization of fluid problems by the lattice Boltzmann method, in IUTAM Symposium on Topological Design Optimization of Structures, Machines and Materials: Status and Perspectives, edited by M.P. Bendsøe, N. Olhoff and O. Sigmund. Springer, Netherlands (2006) 559-568.

[19] A. Evgrafov, G. Pingen and K. Maute, Topology optimization of fluid domains: Kinetic theory approach. Z. Angew. Math. Mech. 88 (2008) 129-141.

[20] A. Evgrafov, K. Maute, R.G. Yang and M.L. Dunn, Topology optimization for nano-scale heat transfer. Int. J. Numer. Methods Engrg. 77 (2009) 285. ISSN 00295981.

[21] R. Eymard, T. Gallouët and R. Herbin, Finite volume methods, in Handbook of Numerical Analysis, edited by P.G. Ciarlet and J.L. Lions 7. North Holland (2000) 713-1020.

[22] R. Eymard, T. Gallouët and R. Herbin, A cell-centred finite-volume approximation for anisotropic diffusion operators on unstructured meshes in any space dimension. IMA J. Numer. Anal 26 (2006) 326-353. http://imajna.oxfordjournals.org/ cgi/content/abstract/26/2/326.

[23] R. Eymard, T. Gallouët, R. Herbin and J.-C. Latche, Analysis tools for finite volume schemes. Acta Math. Univ. Comenianae LXXVI (2007) 111-136.

[24] P. Fernandes, J.M. Guedes and H. Rodrigues, Topology optimization of three-dimensional linear elastic structures with a constraint on "perimeter". Comput. Struct. 73 (1999) 583-594. CODEN CMSTCJ. ISSN 0045-7949.

[25] T. Gallouët, R. Herbin and M.H. Vignal, Error estimates on the approximate finite volume solution of convection diffusion equations with general boundary conditions. SIAM J. Numer. Anal. 37 (2000) 1935-1972. http://link.aip.org/link/?SNA/ $37 / 1935 / 1$

[26] A. Gersborg-Hansen, M. Bendsøe and O. Sigmund, Topology optimization of heat conduction problems using the finite volume method. Struct. Multidisc. Optim. 31 (2006) 251-259. ISSN 1615-147X.

[27] A. Gersborg-Hansen, O. Sigmund and R.B. Haber, Topology optimization of channel flow problems. Struct. Multidisc. Optim. 30 (2005) 181-192.

[28] M.M. Gregersen, F. Okkels, M.Z. Bazant and H. Bruus, Topology and shape optimization of induced-charge electro-osmotic micropumps. New J. Phys. 11 (2009) 075019. http://stacks.iop.org/1367-2630/11/i=7/a=075019.

[29] R.B. Haber, M.P. Bendsøe and C.S. Jog, Perimeter constrained topology optimization of continuum structures, in IUTAM Symposium on Optimization of Mechanical Systems (Stuttgart, 1995). Solid Mech. Appl. 43. Kluwer Acad. Publ., Dordrecht (1996) 113-120.

[30] F.R. Klimetzek, J. Paterson and O. Moos, Autoduct: topology optimization for fluid flow, in Proceedings of Konferenz für angewandte Optimierung. Karlsruhe (2006).

[31] S. Kreissl, G. Pingen, A. Evgrafov and K. Maute, Topology optimization of flexible micro-fluidic devices. Struct. Multidisc. Optim. 42 (2010) 495-516. ISSN 1615-147X. http://dx.doi.org/10.1007/s00158-010-0526-6.

[32] B. Mohammadi and O. Pironneau, Applied shape optimization for fluids. Numerical Mathematics and Scientific Computation, Oxford University Press, New York (2001) xvi+251. ISBN 0-19-850743-7. 
[33] O. Moos, F.R. Klimetzek and R. Rossmann, Bionic optimization of air-guiding systems, in Proceedings of SAE 2004 World Congress \& Exhibition. Detroit, MI, USA, Society of Automotive Engineering, Inc (2004) 95-100.

[34] F. Okkels and H. Bruus, Design of micro-fluidic bio-reactors using topology optimization. J. Comput. Theoret. Nano. 4 (2007) $814-816$.

[35] L.H. Olesen, F. Okkels and H. Bruus, A high-level programming-language implementation of topology optimization applied to steady-state Navier-Stokes flow. Int. J. Numer. Meth. Engrg. 65 (2006) 975-1001.

[36] C. Othmer, A continuous adjoint formulation for the computation of topological and surface sensitivities of ducted flows. Internat. J. Numer. Methods Fluids 58 (2008).

[37] C. Othmer, Th. Kaminski and R. Giering, Computation of topological sensitivities in fluid dynamics: Cost function versatility, in ECCOMAS CFD 2006, Delft (2006).

[38] J. Outrata, M. Kočvara and J. Zowe, Nonsmooth Approach to Optimization Problems with Equilibrium Constraints. Kluwer Academic Publishers, Dordrecht (1998) xxii+273. ISBN 0-7923-5170-3.

[39] J. Petersson, Some convergence results in perimeter-controlled topology optimization. Comput. Methods Appl. Mech. Engrg. 171 (1999) 123-140.

[40] G. Pingen, A. Evgrafov and K. Maute, A parallel Schur complement solver for the solution of the adjoint steady-state lattice Boltzmann equations: application to design optimization. Int. J. Comput. Fluid Dynamics 22 (2008) 464-475.

[41] G. Pingen, A. Evgrafov and K. Maute, Adjoint parameter sensitivity analysis for the hydrodynamic lattice Boltzmann method with applications to design optimization. Comput. Fluids 38 (2009) 910-923.

[42] G. Pingen, M. Waidmann, A. Evgrafov and K. Maute, A parametric level-set approach for topology optimization of flow domains. Struct. Multidisc. Optim. 41 (2010) 117-131. ISSN 1615-147X. http://dx.doi.org/10.1007/s00158-009-0405-1.

[43] K. Svanberg, The method of moving asymptotes - a new method for structural optimization. Int. J. Numer. Methods Engrg. 24 (1987) 359-373. CODEN IJNMBH. ISSN 0029-5981.

[44] K. Svanberg, A class of globally convergent optimization methods based on conservative convex separable approximations. SIAM J. Optim. 12 (2002) 555-573. ISSN 1095-7189.

[45] A.-M. Toader, Convergence of an algorithm in optimal design. Struct. Optim. 13 (1997) 195-198.

[46] E. Wadbro and M. Berggren, Megapixel topology optimization on a graphics processing unit. SIAM Rev. 5 (2009) 707-721.

[47] E. Zeidler, Applied Functional Analysis: Main Principles and Their Applications, 1st edition. Springer (1995). ISBN 0387944222 . 